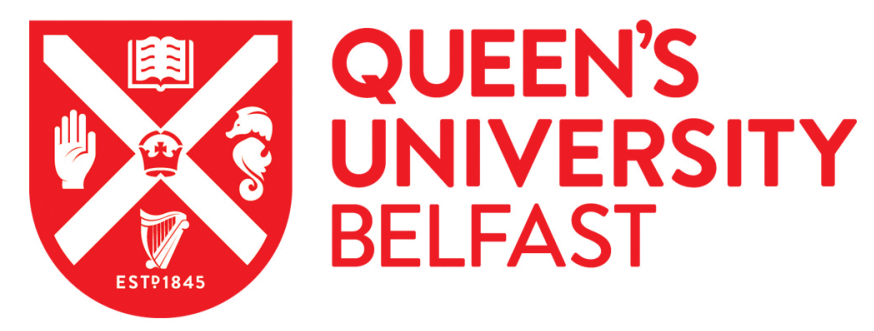

\title{
c-number quantum generalized Langevin equation for an open system
}

Kantorovich, L., Ness, H., Stella, L., \& Lorenz, C. D. (2016). c-number quantum generalized Langevin equation for an open system. Physical Review B, 94(18), [184305]. https://doi.org/10.1103/PhysRevB.94.184305

\author{
Published in: \\ Physical Review B
}

\section{Document Version:}

Peer reviewed version

Queen's University Belfast - Research Portal:

Link to publication record in Queen's University Belfast Research Portal

\section{Publisher rights}

C2016 American Physical Society

This work is made available online in accordance with the publisher's policies.

\section{General rights}

Copyright for the publications made accessible via the Queen's University Belfast Research Portal is retained by the author(s) and / or other copyright owners and it is a condition of accessing these publications that users recognise and abide by the legal requirements associated with these rights.

Take down policy

The Research Portal is Queen's institutional repository that provides access to Queen's research output. Every effort has been made to ensure that content in the Research Portal does not infringe any person's rights, or applicable UK laws. If you discover content in the Research Portal that you believe breaches copyright or violates any law, please contact openaccess@qub.ac.uk. 


\title{
$c$-number Quantum Generalised Langevin Equation for an open system
}

\author{
L. Kantorovich, H. Ness, L. Stella ${ }^{\dagger}$ and C. D. Lorenz \\ Physics Department, King's College London, \\ Strand, London, WC2R 2LS, United Kingdom \\ ${ }^{\dagger}$ Atomistic Simulation Centre, School of Mathematics and Physics, \\ Queen's University Belfast, University Road, \\ Belfast BTr 1NN, Northern Ireland, UK
}

\begin{abstract}
We derive a $c$-number Generalised Langevin Equation (GLE) describing the evolution of the expectation values $\left\langle x_{i}\right\rangle_{t}$ of the atomic position operators $x_{i}$ of an open system. The latter is coupled linearly to a harmonic bath kept at a fixed temperature. The equations of motion contain a non-Markovian friction term with the classical kernel [L. Kantorovich - PRB 78, 094304 (2008)] and a zero mean non-Gaussian random force with correlation functions that depend on the initial preparation of the open system. We used a density operator formalism without assuming that initially the combined system was decoupled. The only approximation made in deriving quantum GLE consists in assuming that the Hamiltonian of the open system at time $t$ can be expanded up to the second order with respect to operators of atomic displacements $u_{i}=x_{i}-\left\langle x_{i}\right\rangle_{t}$ in the open system around their exact atomic positions $\left\langle x_{i}\right\rangle_{t}$ (the "harmonisation" approximation). The noise is introduced to ensure that sampling many quantum GLE trajectories yields exactly the average one. An explicit expression for the pair correlation function of the noise, consistent with the classical limit, is also proposed. Unlike the usually considered quantum operator GLE, the proposed $c$-number quantum GLE can be used in direct molecular dynamic simulations of open systems under general equilibrium or non-equilibrium conditions.
\end{abstract}




\section{INTRODUCTION}

In numerous applications in quantum physics and chemistry [1,2], phenomena of interest are related to an atomistic dynamics of a finite fragment of an extended system. The fragment cannot be treated as isolated as it interacts and exchanges energy with the rest of the system serving as a heat bath. As a complete description of the whole system might be difficult or impossible, one has to look for approaches which pay specific attention to the fragment (an open system), while still retaining the existence of the heat bath. This problem lies within the realm of open quantum dissipative systems [1,2].

In the case of classical systems, molecular dynamics (MD) simulations have proven to be a powerful, yet simple, tool for studying their non-equilibrium properties including tribology [35], energy dissipation [6], crack propagation [7], heat transport [8-14] and irradiation [15]. An appropriate theoretical approach for considering dynamics of open classical systems, based on calculating trajectories of atoms of the open system and accounting for dissipation effects with its environment(s), is provided by the Generalised Langevin Equation (GLE) [16]. Assuming a rather general Hamiltonian of the open system and linear coupling to its harmonic heat bath, one arrives at its non-Markovian classical dynamics with multivariate Gaussian distributed random forces and the memory kernel that is proportional to the random force autocorrelation function (the second fluctuation-dissipation theorem) [17]. Although the GLE has been around for a while (see [1719] and references therein), its application to realistic systems has only recently become realised when a powerful implementation of this method has been proposed $[18,19]$. It solved two main obstacles standing in the way of efficient numerical simulations: its non-Markovian character and the coloured noise. This implementation is also straightforwardly generalised for heat transport simulations which require more than one heat bath [20].

Although classical MD simulations for open systems can be easily justified via GLE, a natural question arises of whether something analogous to GLE can also be formulated in the quantum realm. By that we mean equations of motion for the expectation values of positions of atoms in the open system, $\left\langle x_{i}\right\rangle_{t}=\operatorname{Tr}\left(\rho(t) x_{i}\right)$ (where $\rho(t)$ is the exact density matrix at time $t$ of the whole combined system, and $x_{i}$ the operator of the coordinate of atom $i$ ); the equations are expected to contain a non-operator (or $c$-number) stochastic force with certain statistical properties. At high enough temperatures (or in the $\hbar \rightarrow 0$ limit) the $c$-number quantum GLE must coincide with the classical one for the same Hamiltonian [17]. Using such a tool, one will be able to study, via MD-like approaches, dynamical phenomena of a wide range of systems e.g. in quantum optics, condensed 
matter and chemical physics and nanotechnology, accounting for the full quantum nature of both the bath and the open system.

First attempts to develop a quantum analogue of the classical GLE based on equations of motion for Heisenberg positions and momenta operators of atoms of an open system were done by Ford, Kac and Mazur (FKM) [21]. This method has been further developed by other authors [22-33] and then applied, in particular, to heat transport [10, 34, 35]. In this method, GLE-like non-Markovian equations for operators of the open system are obtained assuming a linear coupling to the harmonic bath. The bath enters these equations via an operator which has a meaning of a force, the latter contains a linear combination of initial bath operators. Assuming that initially the bath was at equilibrium at a certain temperature, and hence the reduced density matrix of the bath (the density matrix of the whole system in which open system degrees of freedom are traced out) is canonical at a certain temperature, one can show that the statistically averaged operator of the force is zero and its correlation function is essentially given by the well-known displacement-displacement correlation function calculated in the harmonic approximation. Although this method is exact within the adopted Hamiltonian, analytical solutions can only be obtained in simple cases [32]. It is essential, that even though the equations themselves are written only for operators of the open system, these operators are still defined in the Hilbert space of the whole system (open system + bath). For harmonic systems, this approach for heat transport has been shown [10] to be fully equivalent to the method based on non-equilibrium Green's functions [36-38].

Note that equations for quantum operators of an open system with linearly coupled harmonic bath can also be derived using path-integral techniques by integrating out the bath variables [2, 3942]. This method is however based on the so-called partitioned assumption (initiated by Feynman and Vernon [39]) that the initial density matrix is a direct product of independent density matrices of the open system and bath (the Born approximation [1]) .

Several attempts have also been made to obtain truly $c$-number quantum GLE (cQGLE). In a hybrid approach $[43,44]$ the authors suggested simply to replace, without proper justification, Heisenberg equations of motion for operators of positions of atoms of an open system by their classical analogues keeping, at the same time, the fully quantum expression for the random force autocorrelation function (note that in this approach the random force is not an operator).

A more elaborate approach based on a somewhat artificial distribution function for the bath has also been proposed [45-48]. The corresponding ansatz of a coherent state representation provides a connection with the classical GLE in the limit of $\hbar \rightarrow 0$. However, some ad hoc, not fully justifiable, 
assumptions are used for the form of the quantum Hamiltonian and the way the quantum thermal averages are performed [49].

We also note that in most of the methods mentioned above the bath Hamiltonian was represented as a set of independent harmonic oscillators.

Here we offer a fully consistent derivation of cQGLE for an open system based on the density matrix method. The Born approximation for the initial density matrix of the whole system is not used, i.e. the whole system initially is not assumed to be partitioned. We consider a general Hamiltonian for the open system which is linearly coupled to the harmonic bath. One of the advantages of our model is that, similarly to our classical treatment [17-19], the bath and the open system are treated as parts of the same whole system. We show that, using a plausible so-called "harmonisation" approximation, a class of cQGLEs for the mean values $\left\langle x_{i}\right\rangle_{t}$ of atoms in the open quantum system can be established. These equations are non-Markovian in nature with a friction kernel which is identical to that found in the classical GLE [17], while the random force, contrary to the classical case, is non-Gaussian. It is shown to have a zero mean with the pair correlation function being of the same functional form as in the classical case. Next, we establish a connection with the previously developed methods and obtain an explicit expression for the pair correlation function of the random force by assuming that the order in which the stochastic and quantum-mechanical averages are performed must not affect the final result.

\section{HAMILTONIAN AND EXACT HEISENBERG EQUATIONS OF MOTION FOR OP- ERATORS}

Consider a system consisting of two parts: a finite open system (or region 1) and an infinite heat bath (region 2). Correspondingly, subscripts 1 and 2 will be used in vectors and matrices, where appropriate. We assume that the heat bath is much larger than the system itself and hence can be asigned to have a fixed temperature $T$. The Hamiltonian of the whole system, $\mathcal{H}=\mathcal{H}_{1}+\mathcal{H}_{2}+\mathcal{H}_{12}$, contains the Hamiltonian of the open system,

$$
\mathcal{H}_{1}=\sum_{i \in 1} \frac{p_{i}^{2}}{2 m_{i}}+W\left(\mathbf{x}_{1}\right)=\frac{1}{2} \mathbf{p}_{1}^{T} \mathbf{M}_{11}^{-1} \mathbf{p}_{1}+W\left(\mathbf{x}_{1}\right)
$$

which assumes an arbitrary potential energy term, $W\left(\mathbf{x}_{1}\right)$, the harmonic bath,

$$
\mathcal{H}_{2}=\frac{1}{2} \sum_{i, j \in 2}\left(\frac{q_{i}^{2}}{m_{i}} \delta_{i j}+\Phi_{i j} u_{i} u_{j}\right)=\frac{1}{2} \mathbf{q}_{2}^{T} \mathbf{M}_{22}^{-1} \mathbf{q}_{2}+\frac{1}{2} \mathbf{u}_{2}^{T} \boldsymbol{\Phi}_{22} \mathbf{u}_{2}
$$


and the interaction between the system and bath regions which is assumed to be linear with respect to atomic displcements $u_{k}$ of the bath atoms:

$$
\mathcal{H}_{12}=\sum_{i \in 2} h_{i} u_{i}=\mathbf{h}_{2}^{T} \mathbf{u}_{2}
$$

Here $\mathbf{x}_{1}=\left(x_{i}\right)$ and $\mathbf{p}_{1}=\left(p_{i}\right)$ are vector-columns of all Cartesian coordinates $i$ of the atoms in the system and their momenta, respectively; $\mathbf{u}_{2}=\left(u_{i}\right)$ and $\mathbf{q}_{2}=\left(q_{i}\right)$ are vector-columns of all atomic displacements in the bath and their corresponding momentum operators. $\boldsymbol{\Phi}_{22}=\left(\Phi_{i j}\right)$ is the bath force-constant matrix, and $\mathbf{M}_{11}=\left(\delta_{i j} m_{i}\right)$ and $\mathbf{M}_{22}=\left(\delta_{i j} m_{i}\right)$ are the diagonal matrices of atomic masses of the system and bath, respectively. The superscript $T$ means transpose. The vector $\mathbf{h}_{2}=\left(h_{i}\left(\mathbf{x}_{1}\right)\right)$ defines (minus) forces with which atoms in the system act on the atoms of the bath; this vector is assumed to have an arbitrary dependence on $\mathbf{x}_{1}$.

The above Hamiltonian is exactly the same as in the recent classical formulation of the GLE equations [17]. It is more general than the Caldeira-Leggett Hamiltonian [50] containing independent harmonic oscillators in the bath and the coupling which is linear in both bath and system coordinates (the bilinear coupling), that is normally used in quantum theories of GLE [2, 2123, 25, 32, 35, 43]. Our Hamiltonian can be obtained from the full Hamiltonian of the whole combined system by expanding it until the second order in terms of atomic displacements in the bath. Hence, its parameters can be taken directly from the adopted Hamiltonian of the realistic system under study [19]. Note that $\mathcal{H}_{1}$ includes the interaction between atoms in the system and their counterparts in the bath which are clumped at their equilibrium positions; any variation of the system-bath interaction due to the bath atoms vibrating around these positions is accounted for by the rest of the combined bath Hamiltonian $\mathcal{H}_{b}=\mathcal{H}_{2}+\mathcal{H}_{12}$.

Next we introduce the density matrix operator, $\rho(t)$, for the system and bath, which satisfies the appropriate Liouville equation with the full Hamiltonian. We recall [51] that the general solution of the Liouville equation for time-independent Hamiltonian $\mathcal{H}$ is $\rho(t)=U \rho^{0} U^{\dagger}$, where $U \equiv U\left(t, t_{0}\right)$ and $U\left(t_{1}, t_{2}\right)=\exp \left[-i \mathcal{H}\left(t_{1}-t_{2}\right) / \hbar\right]$ is the evolution operator, and $\rho^{0}$ is the density matrix at the initial time $t_{0}$. Correspondingly, an operator $A$ in the Heisenberg picture (to be denoted with the tilda in the following), $\widetilde{A}(t)=U^{\dagger} A U$, satisfies the equation of motion $i \hbar \partial_{t} \widetilde{A}(t)=U^{\dagger}[A, \mathcal{H}] U$.

Our goal is to obtain a closed set of equations for the expectation values of the atomic positions, $\left\langle x_{i}\right\rangle_{t}=\operatorname{Tr}\left(\rho(t) x_{i}\right)=\operatorname{Tr}\left(\rho_{0} \widetilde{x}_{i}(t)\right)$ for $i \in 1$, by eliminating the degrees of freedom of the bath atoms. Ideally, we would like these equations to resemble classical GLE with a friction memory term and stochastic forces. To this end, instead of the operators of the bath $\mathbf{u}_{2}$ and $\mathbf{q}_{2}$, it is convenient to introduce their mass-scaled counterparts $\mathbf{x}_{2}=\left(x_{i} ; i \in 2\right)=\mathbf{M}_{22}^{1 / 2} \mathbf{u}_{2}$ and $\mathbf{p}_{2}=\left(p_{i} ; i \in 2\right)=$ 
$\mathbf{M}_{22}^{-1 / 2} \mathbf{q}_{2}$, which satisfy the same commutation relations, $\left[x_{i}, p_{j}\right]=i \hbar \delta_{i j}(i, j \in 2)$. Then the combined bath and interaction Hamiltonian takes on the following form:

$$
\mathcal{H}_{b}=\mathcal{H}_{12}+\mathcal{H}_{2}=\frac{1}{2} \mathbf{p}_{2}^{T} \mathbf{p}_{2}+\frac{1}{2} \mathbf{x}_{2}^{T} \mathbf{D}_{22} \mathbf{x}_{2}+\mathbf{V}_{2}^{T} \mathbf{x}_{2}
$$

where $\mathbf{V}_{2}=\mathbf{h}_{2} \mathbf{M}_{22}^{-1 / 2}$ are the appropriately rescaled coefficients explicitly depending on $\mathbf{x}_{1}$.

By calculating commutators of the operators of coordinates and momenta of both regions $\left(\mathbf{x}_{1}\right.$, $\mathbf{x}_{2}, \mathbf{p}_{1}$ and $\mathbf{p}_{2}$ ) with the Hamiltonian $\mathcal{H}$, the equations of motion for the operators $\widetilde{\mathbf{x}}_{1}, \widetilde{\mathbf{x}}_{2}, \widetilde{\mathbf{p}}_{1}$ and $\widetilde{\mathbf{p}}_{2}$ in the Heisenberg representation are obtained. For the system we have:

$$
\mathbf{M}_{11} \partial_{t} \widetilde{\mathbf{x}}_{1}=\widetilde{\mathbf{p}}_{1}, \quad \partial_{t} \widetilde{\mathbf{p}}_{1}=-\mathbf{h}_{1}\left(\widetilde{\mathbf{x}}_{1}\right)-\mathbf{V}_{12}\left(\widetilde{\mathbf{x}}_{1}\right) \widetilde{\mathbf{x}}_{2} \equiv \mathbf{M}_{11} \partial_{t}^{2} \widetilde{\mathbf{x}}_{1}
$$

where $\mathbf{h}_{1}\left(\mathbf{x}_{1}\right)=\partial W / \partial \mathbf{x}_{1}=\left(\partial W / \partial x_{i} ; i \in 1\right)$ and

$$
\mathbf{V}_{12}\left(x_{1}\right)=\frac{\partial \mathbf{V}_{2}\left(\mathbf{x}_{1}\right)}{\partial \mathbf{x}_{1}}=\left(\frac{1}{\sqrt{m_{j}}} \frac{\partial h_{j}\left(\mathbf{x}_{1}\right)}{\partial x_{i}} ; i \in 1, j \in 2\right)
$$

and hence $\mathbf{h}_{1}\left(\widetilde{\mathbf{x}}_{1}\right)=U^{\dagger}\left(\partial W / \partial \mathbf{x}_{1}\right) U=\partial W\left(\widetilde{\mathbf{x}}_{1}\right) / \partial \widetilde{\mathbf{x}}_{1}$ and $\mathbf{V}_{12}\left(\widetilde{\mathbf{x}}_{1}\right)=\partial \mathbf{V}_{2}\left(\widetilde{\mathbf{x}}_{1}\right) / \partial \widetilde{\mathbf{x}}_{1}$.

Similarly for the bath:

$$
\partial_{t} \widetilde{\mathbf{x}}_{2}=\widetilde{\mathbf{p}}_{2}, \quad \partial_{t} \widetilde{\mathbf{p}}_{2}=-\mathbf{D}_{22} \widetilde{\mathbf{x}}_{2}-\mathbf{V}_{2}\left(\widetilde{\mathbf{x}}_{1}\right) \equiv \partial_{t}^{2} \widetilde{\mathbf{x}}_{2}
$$

where $\mathbf{D}_{22}=\mathbf{M}_{22}^{-1 / 2} \boldsymbol{\Phi}_{22} \mathbf{M}_{22}^{-1 / 2}$ is the dynamical matrix of the bath.

The equations (II.6) for the coordinates $\widetilde{\mathbf{x}}_{2}(t)$ of the bath atoms are solved in exactly the same way as in the classical case [17] by first defining normal coordinates $\xi_{\lambda}=\sum_{i \in 2} e_{\lambda i} x_{i}=\mathbf{e}_{\lambda}^{T} \mathbf{x}_{1}$ of the bath expressed via the eigenvectors $\mathbf{e}_{\lambda}$ of the dynamical matrix, $\mathbf{D}_{22} \mathbf{e}_{\lambda}=\omega_{\lambda}^{2} \mathbf{e}_{\lambda}$, where $\omega_{\lambda}$ are frequencies of the bath's normal vibrational modes. In the new coordinates we obtain decoupled differential equations for each normal mode $\lambda$ as $\ddot{\widetilde{\xi}}_{\lambda}+\omega_{\lambda}^{2} \widetilde{\xi}_{\lambda}=-V_{\lambda}(t)$ (dots above the symbols denote time derivatives), where $V_{\lambda}(t)=\mathbf{e}_{\lambda}^{T} \mathbf{V}_{2}$, so that their solutions are readily obtained $(i \in 2)$ :

$$
\begin{gathered}
\widetilde{x}_{i}(t)=\sum_{\lambda} e_{\lambda i} \widetilde{\xi}_{\lambda}(t)=\sum_{\lambda} e_{\lambda i}\left[A_{\lambda} e^{i \omega_{\lambda} t}+B_{\lambda} e^{-i \omega_{\lambda} t}-\frac{1}{\omega_{\lambda}} \int_{t_{0}}^{t} V_{\lambda}(\tau) \sin \left[\omega_{\lambda}(t-\tau)\right] d \tau\right] \\
\widetilde{p}_{i}(t)=\sum_{\lambda} e_{\lambda i} \dot{\widetilde{\xi}}_{\lambda}(t)=\sum_{\lambda} e_{\lambda i}\left[i \omega_{\lambda}\left(A_{\lambda} e^{i \omega_{\lambda} t}-B_{\lambda} e^{-i \omega_{\lambda} t}\right)-\int_{t_{0}}^{t} V_{\lambda}(\tau) \cos \left[\omega_{\lambda}(t-\tau)\right] d \tau\right]
\end{gathered}
$$

where $A_{\lambda}$ and $B_{\lambda}$ are two operators to be determined from the initial conditions: $\widetilde{x}_{i}\left(t_{0}\right)=x_{i}$ and $\tilde{p}_{i}\left(t_{0}\right)=p_{i}$. Some simple algebra yields the following expression for the (rescaled) atomic positions of the bath atoms:

$$
\widetilde{\mathbf{x}}_{2}(t)=\dot{\Omega}_{22}\left(t-t_{0}\right) \mathbf{x}_{2}+\Omega_{22}\left(t-t_{0}\right) \mathbf{p}_{2}-\int_{t_{0}}^{t} \Omega_{22}(t-\tau) \mathbf{V}_{2}(\tau) d \tau
$$


where

$$
\Omega_{22}(t)=\sum_{\lambda} \frac{\mathbf{e}_{\lambda} \mathbf{e}_{\lambda}^{T}}{\omega_{\lambda}} \sin \left(\omega_{\lambda} t\right)
$$

and

$$
\dot{\Omega}_{22}(t)=\sum_{\lambda} \mathbf{e}_{\lambda} \mathbf{e}_{\lambda}^{T} \cos \left(\omega_{\lambda} t\right)
$$

are two square bath matrices (cf. [17]). The time integral in Eq. (II.7) can be calculated by parts. Defining one more bath matrix [17]

$$
\boldsymbol{\Pi}_{22}(t)=\sum_{\lambda} \frac{\mathbf{e}_{\lambda} \mathbf{e}_{\lambda}^{T}}{\omega_{\lambda}^{2}} \cos \left(\omega_{\lambda} t\right)
$$

and noticing that $\mathbf{D}_{22}^{-1}=\sum_{\lambda} \omega_{\lambda}^{-2} \mathbf{e}_{\lambda} \mathbf{e}_{\lambda}^{T} \equiv \Pi_{22}(0)$, we obtain:

$$
\begin{gathered}
\widetilde{\mathbf{x}}_{2}(t)=\left[\dot{\boldsymbol{\Omega}}_{22}\left(t-t_{0}\right) \mathbf{x}_{2}+\boldsymbol{\Omega}_{22}\left(t-t_{0}\right) \mathbf{p}_{2}+\boldsymbol{\Pi}_{22}\left(t-t_{0}\right) \mathbf{V}_{2}\left(t_{0}\right)\right]-\mathbf{D}_{22}^{-1} \mathbf{V}_{2}(t) \\
+\int_{t_{0}}^{t} \boldsymbol{\Pi}_{22}(t-\tau)\left[\frac{d}{d \tau} \mathbf{V}_{2}(\tau)\right] d \tau
\end{gathered}
$$

The time derivative of the operator $\mathbf{V}_{2}(\tau)$ is

$$
\partial_{\tau} \mathbf{V}_{2}(\tau)=\frac{1}{i \hbar} U^{\dagger}\left[\mathbf{V}_{2}\left(\mathbf{x}_{1}\right), \mathcal{H}\right] U=\frac{1}{i \hbar} U^{\dagger}\left[\mathbf{V}_{2}\left(\mathbf{x}_{1}\right), \frac{1}{2} \mathbf{p}_{1}^{T} \mathbf{M}_{11}^{-1} \mathbf{p}_{1}\right] U=\mathbf{V}_{21}\left(\mathbf{x}_{1}\right) \partial_{\tau} \widetilde{\mathbf{x}}_{1}(\tau)-\frac{i \hbar}{2} \overline{\mathbf{V}}_{2}(\tau)
$$

where $\overline{\mathbf{V}}_{2}(\tau)=\left(\bar{V}_{j}(\tau) ; j \in 2\right)$ with $\bar{V}_{j}(\tau)=\sum_{i \in 1} m_{i}^{-1} \partial^{2} V_{j}\left(\widetilde{\mathbf{x}}_{1}\right) / \partial \widetilde{x}_{i}^{2}$. Note that the last term in Eq. (II.11) vanishes in the $\hbar \rightarrow 0$ limit. As we shall see immediately, it will be responsible for a contribution to the force which does not have the form of the friction force. Correspondingly,

$$
\begin{aligned}
\widetilde{\mathbf{x}}_{2}(t) & =\left[\dot{\boldsymbol{\Omega}}_{22}\left(t-t_{0}\right) \mathbf{x}_{2}+\boldsymbol{\Omega}_{22}\left(t-t_{0}\right) \mathbf{p}_{2}+\boldsymbol{\Pi}_{22}\left(t-t_{0}\right) \mathbf{V}_{2}\left(t_{0}\right)\right]-\mathbf{D}_{22}^{-1} \mathbf{V}_{2}(t) \\
& +\int_{t_{0}}^{t} \boldsymbol{\Pi}_{22}(t-\tau) \mathbf{V}_{21}(\tau) \partial_{\tau} \widetilde{\mathbf{x}}_{1}(\tau) d \tau-\frac{i \hbar}{2} \int_{t_{0}}^{t} \boldsymbol{\Pi}_{22}(t-\tau) \overline{\mathbf{V}}_{2}(\tau) d \tau
\end{aligned}
$$

Substituting this expression into the equation of motion (II.5) for the system atoms, we obtain the quantum GLE (the differential equation for Heisenberg position operators of the open system):

$$
\begin{gathered}
\mathbf{M}_{11} \ddot{\widetilde{\mathbf{x}}}_{1}(t)=-\mathbf{h}_{1}(t)+\mathbf{V}_{12}(t) \mathbf{D}_{22}^{-1} \mathbf{V}_{2}(t)+\mathbf{R}_{1}(t) \\
-\int_{t_{0}}^{t} \mathbf{K}_{11}(t, \tau) \dot{\widetilde{\mathbf{x}}}_{1}(\tau) d \tau+\frac{i \hbar}{2} \int_{t_{0}}^{t} \mathbf{V}_{12}(t) \boldsymbol{\Pi}_{22}(t-\tau) \overline{\mathbf{V}}_{2}(\tau) d \tau
\end{gathered}
$$

where

$$
\mathbf{K}_{11}(t, \tau)=\mathbf{V}_{12}(t) \boldsymbol{\Pi}_{22}(t-\tau) \mathbf{V}_{21}(\tau)
$$




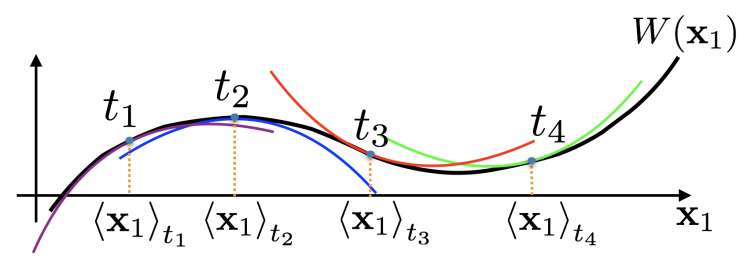

Figure 1. In the harmonisation approximation a general potential function $W\left(\mathbf{x}_{1}\right)$ (black) of the coordinates $\mathbf{x}_{1}$ is approximated by parabolas at different times (coloured curves) which are fit around the reference atomic positions $\mathbf{x}_{1}^{\mathrm{ref}}(t)$. The latter, after the dynamics for the harmonisation approximation is calculated exactly, are taken to be the quantum averages $\left\langle\mathbf{x}_{1}\right\rangle_{t}$ themselves, i.e. the instantaneous positions of the atoms at time $t$.

is the friction kernel (cf. [17]) and

$$
\mathbf{R}_{1}(t)=-\mathbf{V}_{12}(t)\left[\dot{\Omega}_{22}\left(t-t_{0}\right) \mathbf{x}_{2}+\boldsymbol{\Omega}_{22}\left(t-t_{0}\right) \mathbf{p}_{2}+\boldsymbol{\Pi}_{22}\left(t-t_{0}\right) \mathbf{V}_{2}\left(t_{0}\right)\right]
$$

The obtained (operator) quantum GLE is exact for the Hamiltonian we adopted. We first note that the first integral term in the GLE (II.12) has exactly the same form as the classical friction force [17]; however, there is also a second integral term, which does not have the form of a friction force. This is a purely "non-classical" term as it disappears in the classical limit of $\hbar \rightarrow 0$. More importantly, the obtained GLE is highly non-linear with respect to the Heisenberg positions $\widetilde{\mathbf{x}}_{1}(t)$ as $\mathbf{h}_{1}, \mathbf{V}_{2}, \mathbf{V}_{21}$ and $\overline{\mathbf{V}}_{2}$ all depend on it (and hence on time). Therefore, when multiplying both sides of this equation by the initial density matrix $\rho^{0}$ and taking the trace over the Hilbert space of the whole combined system, it will not be possible to obtain a self-containing equation for the averages $\left\langle\mathbf{x}_{1}\right\rangle_{t}$. This is because the average of a function is not generally equal to the function of the average, e.g. $\left\langle\mathbf{h}_{1}\left(\widetilde{\mathbf{x}}_{1}\right)\right\rangle_{t}=\operatorname{Tr}\left[\rho^{0} \mathbf{h}_{1}\left(\widetilde{\mathbf{x}}_{1}\right)\right] \neq \mathbf{h}_{1}\left(\left\langle\mathbf{x}_{1}\right\rangle_{t}\right)$. Only for linear operators $\mathbf{h}_{1}\left(x_{1}\right)=\mathbf{h}_{1}^{0}+\mathbf{h}_{11}^{0} \mathbf{x}_{1}$ we would have the equality, $\left\langle\mathbf{h}_{1}\left(\widetilde{\mathbf{x}}_{1}\right)\right\rangle_{t}=\mathbf{h}_{1}\left(\left\langle\mathbf{x}_{1}\right\rangle_{t}\right)$. Therefore, there is a certain difficulty in formulating a self-contained $c$-number quantum GLE in this rather general case. This situation is fully resolved within the harmonisation approximation to be introduced next.

\section{QUANTUM GLE WITHIN THE HARMONISATION APPROXIMATION}

In order to obtain a closed set of equations for the expectation values of the atomic positions, $\left\langle x_{i}\right\rangle_{t}$ for all $i \in 1$, we shall expand all operators in the Hamiltonian into a power series of the displacement operators. To explain the meaning of this kind of expansion, consider an arbitrary 
operator $A\left(\mathbf{x}_{1}\right)$ that explicitly depends on the atomic positions of atoms in region 1 . Introducing displacements operators $u_{i}=x_{i}-x_{i}^{\text {ref }}$ for all atoms $i \in 1$, calculated with respect to some (in general, arbitrary) reference atomic positions, $\mathbf{x}_{1}^{\text {ref }}(t)$, which are not operators (i.e. are $c$-numbers) and may also depend on time, one can expand the operator $A$ in a Maclaurin series as follows:

$$
A\left(\mathbf{x}_{1}\right)=A^{0}+\sum_{i \in 1} A_{i}^{0} u_{i}+\sum_{i, j \in 1} A_{i j}^{0} u_{i} u_{j}+\ldots
$$

where $A^{0}=A\left(\mathbf{x}_{1}^{\text {ref }}\right)$ and the linear and second order expansion coefficients,

$$
A_{i}^{0}=\frac{\partial A\left(\mathbf{x}_{1}^{\mathrm{ref}}\right)}{\partial x_{i}^{\mathrm{ref}}} \quad \text { and } \quad A_{i j}^{0}=\frac{\partial^{2} A\left(\mathbf{x}_{1}^{\mathrm{ref}}\right)}{\partial x_{i}^{\mathrm{ref}} \partial x_{j}^{\mathrm{ref}}}
$$

are not operators, but rather real functions of the reference atomic positions. Since the latter may depend on time, the expansion coefficients will also be time dependent.

In our case only the terms $W\left(\mathbf{x}_{1}\right)$ and $\mathbf{h}_{2}\left(\mathbf{x}_{1}\right)$ in the components $\mathcal{H}_{1}$ and $\mathcal{H}_{12}$ of the Hamiltonian, respectively, depend on the operators $\mathbf{x}_{1}$ of the atomic positions. We expand these terms up to the second order in the displacements $u_{i}=x_{i}-x_{i}^{\text {ref }}$. This method, which we shall call a harmonisation approximation, is inspired by the expansion method used in the CEID theory [52, 53]. We will see later that there is an obvious and straightforward way to choose the reference atomic positions, $\mathbf{x}_{1}^{\mathrm{ref}}(t)$; until then, they remain arbitrary.

In the harmonisation approximation (HA) we obtain:

$$
\begin{gathered}
\mathcal{H}_{1} \simeq \mathcal{H}_{1}^{\mathrm{HA}}(t)=\frac{1}{2} \mathbf{p}_{1}^{T} \mathbf{M}_{11}^{-1} \mathbf{p}_{1}+W(t)+\mathbf{h}_{1}^{T}(t) \mathbf{u}_{1}+\frac{1}{2} \mathbf{u}_{1}^{T} \boldsymbol{\Phi}_{11}(t) \mathbf{u}_{1} \\
\mathcal{H}_{12} \simeq \mathcal{H}_{12}^{\mathrm{HA}}(t)=\mathbf{h}_{2}^{T}(t) \mathbf{u}_{2}+\mathbf{u}_{1}^{T} \mathbf{h}_{12}(t) \mathbf{u}_{2}
\end{gathered}
$$

where the expansion coefficients are: $W(t)=W\left(\mathbf{x}_{1}^{\mathrm{ref}}\right), \mathbf{h}_{2}(t)=\left(h_{i}\left(\mathbf{x}_{1}^{\mathrm{ref}}\right) ; i \in 2\right)$,

$$
\begin{gathered}
\mathbf{h}_{1}(t)=\left(h_{i} ; i \in 1\right)=\frac{\partial W\left(\mathbf{x}_{1}^{\mathrm{ref}}\right)}{\partial \mathbf{x}_{1}^{\mathrm{ref}}} \quad \text { with } \quad h_{i}=\frac{\partial W\left(\mathbf{x}_{1}^{\mathrm{ref}}\right)}{\partial x_{i}^{\mathrm{ref}}}, \\
\boldsymbol{\Phi}_{11}(t)=\left(\Phi_{i j} ; i, j \in 1\right)=\frac{\partial^{2} W\left(\mathbf{x}_{1}^{\mathrm{ref}}\right)}{\partial \mathbf{x}_{1}^{\mathrm{ref}} \partial \mathbf{x}_{1}^{\mathrm{ref}}} \quad \text { with } \quad \Phi_{i j}=\frac{\partial^{2} W\left(\mathbf{x}_{1}^{\mathrm{ref}}\right)}{\partial x_{i}^{\mathrm{ref}} \partial x_{j}^{\mathrm{ref}}}
\end{gathered}
$$

and

$$
\mathbf{h}_{12}(t)=\left(h_{i j} ; i \in 1, j \in 2\right)=\frac{\partial \mathbf{h}_{2}\left(\mathbf{x}_{1}^{\mathrm{ref}}\right)}{\partial \mathbf{x}_{1}^{\mathrm{ref}}} \quad \text { with } \quad h_{i j}=\frac{\partial h_{j}\left(\mathbf{x}_{1}^{\mathrm{ref}}\right)}{\partial x_{i}^{\mathrm{ref}}}
$$

All of the above expressions are ordinary derivatives of the interaction $W$ and the (minus) forces $h_{2}$, both being $c$-numbers, i.e. they are to be understood as real functions of the reference atomic positions $\mathbf{x}_{1}^{\mathrm{ref}}(t)$. Note that all these coefficients are explicit functions of time; they are not 
operators and can be easily calculated given the explicit functional dependences in $W\left(\mathbf{x}_{1}\right)$ and $\mathbf{h}_{2}\left(\mathbf{x}_{1}\right)$.

Next, the equations of motion for the operators need to be obtained. The method of the previous section can be applied here with the caviat that now, after the harmonisation approximation, the Hamiltonian depends on time explicitly, $\mathcal{H}(t)=\mathcal{H}_{1}^{\mathrm{HA}}(t)+\mathcal{H}_{12}^{\mathrm{HA}}(t)+\mathcal{H}_{2}$, see Eqs. (III.3) and (III.4). Hence the time evolution of the density matrix and the Heisenberg representation of operators are to be obtained using a more general evolution operator

$$
U\left(t, t_{0}\right)=\widehat{T} \exp \left(-\frac{i}{\hbar} \int_{t_{0}}^{t} \mathcal{H}(\tau) d \tau\right)
$$

where $\widehat{T}$ is the time-ordering operator (assuming $t>t_{0}$ ). The method of obtaining the equations of motion for the operators is still straightforward as it requires the calculation of the commutators of the operators $\mathbf{x}_{1}, \mathbf{p}_{1}, \mathbf{q}_{2}$ and $\mathbf{u}_{2}$ with the Hamiltonian. Note that from now on, the dynamics of the atoms in the open system are calculated with respect to the Hamiltonian in the harmonisation approximation.

Therefore, introducing again the rescaled variables for the bath atoms, the combined bath and interaction Hamiltonian takes on a simpler form:

$$
\mathcal{H}_{b}=\mathcal{H}_{12}^{\mathrm{HA}}+\mathcal{H}_{2}=\frac{1}{2} \mathbf{p}_{2}^{T} \mathbf{p}_{2}+\frac{1}{2} \mathbf{x}_{2}^{T} \mathbf{D}_{22} \mathbf{x}_{2}+\left(\mathbf{V}_{2}+\mathbf{V}_{21} \mathbf{u}_{1}\right)^{T} \mathbf{x}_{2}
$$

where $\mathbf{V}_{2}=\mathbf{h}_{2} \mathbf{M}_{22}^{-1 / 2}$ and $\mathbf{V}_{12}=\mathbf{V}_{21}^{T}=\mathbf{h}_{12} \mathbf{M}_{22}^{-1 / 2}$ are the appropriately rescaled coefficients (which depend on the reference positions $\mathbf{x}_{1}^{\text {ref }}$ ). Calculating the commutators with $\mathcal{H}$, the following equations of motion are obtained for the operators in the Heisenberg picture:

$$
\mathbf{M}_{11} \partial_{t} \widetilde{\mathbf{x}}_{1}=\widetilde{\mathbf{p}}_{1}, \quad \partial_{t} \widetilde{\mathbf{p}}_{1}=-\mathbf{h}_{1}-\mathbf{V}_{12} \widetilde{\mathbf{x}}_{2}-\mathbf{\Phi}_{11} \widetilde{\mathbf{u}}_{1} \equiv \mathbf{M}_{11} \partial_{t}^{2} \widetilde{\mathbf{x}}_{1}
$$

where $\widetilde{\mathbf{u}}_{1}=\widetilde{\mathbf{x}}_{1}-\mathbf{x}_{1}^{\text {ref }}$, and

$$
\partial_{t} \widetilde{\mathbf{x}}_{2}=\widetilde{\mathbf{p}}_{2}, \quad \partial_{t} \widetilde{\mathbf{p}}_{2}=-\mathbf{D}_{22} \widetilde{\mathbf{x}}_{2}-\left(\mathbf{V}_{2}+\mathbf{V}_{21} \widetilde{\mathbf{u}}_{1}\right) \equiv \partial_{t}^{2} \widetilde{\mathbf{x}}_{2}
$$

The latter equation results in the decoupled differential equations for each normal mode $\lambda$ as $\ddot{\widetilde{\xi}}_{\lambda}+\omega_{\lambda}^{2} \widetilde{\xi}_{\lambda}=-V_{\lambda}(t)$, where this time $V_{\lambda}(t)=\mathbf{e}_{\lambda}^{T}\left(\mathbf{V}_{2}+\mathbf{V}_{21} \widetilde{\mathbf{u}}_{1}\right)$. Note that the non-linear term we encountered in Eq. (II.11) in the previous Section does not appear here, i.e. it is absent within the harmonisation approximation.

Then, the equations for the bath are easily solved similarly to the general case considered in the previous section:

$$
\widetilde{\mathbf{x}}_{2}(t)=\dot{\Omega}_{22}\left(t-t_{0}\right) \mathbf{x}_{2}+\Omega_{22}\left(t-t_{0}\right) \mathbf{p}_{2}-\int_{t_{0}}^{t} \Omega_{22}(t-\tau)\left[\mathbf{V}_{2}(\tau)+\mathbf{V}_{21}(\tau) \widetilde{\mathbf{u}}_{1}(\tau)\right] d \tau
$$


Noticing that $\partial_{\tau} \mathbf{V}_{2}(\tau)=\partial_{\tau} \mathbf{V}_{2}\left(\mathbf{x}_{1}^{\mathrm{ref}}(\tau)\right)=\mathbf{V}_{21} \partial_{\tau} \mathbf{x}_{1}^{\mathrm{ref}}(\tau)$ and $\partial_{\tau} \widetilde{\mathbf{u}}_{1}(\tau)=\partial_{\tau} \widetilde{\mathbf{x}}_{1}(\tau)-\partial_{\tau} \mathbf{x}_{1}^{\mathrm{ref}}(\tau)$, the integral above is calculated by parts to yield:

$$
\begin{gathered}
\widetilde{\mathbf{x}}_{2}(t)=\left[\dot{\boldsymbol{\Omega}}_{22}\left(t-t_{0}\right) \mathbf{x}_{2}+\boldsymbol{\Omega}_{22}\left(t-t_{0}\right) \mathbf{p}_{2}+\boldsymbol{\Pi}_{22}\left(t-t_{0}\right) \mathbf{g}_{2}\right] \\
-\mathbf{D}_{22}^{-1}\left[\mathbf{V}_{2}(t)+\mathbf{V}_{21}(t) \widetilde{\mathbf{u}}_{1}(t)\right]+\int_{t_{0}}^{t} \boldsymbol{\Pi}_{22}(t-\tau)\left[\dot{\mathbf{V}}_{21}(\tau) \widetilde{\mathbf{u}}_{1}(\tau)+\mathbf{V}_{21}(\tau) \dot{\tilde{\mathbf{x}}}_{1}(\tau)\right] d \tau
\end{gathered}
$$

where

$$
\mathbf{g}_{2}=\mathbf{V}_{2}\left(t_{0}\right)+\mathbf{V}_{21}\left(t_{0}\right) \mathbf{u}_{1}
$$

and $\mathbf{u}_{1}=\widetilde{\mathbf{u}}\left(t_{0}\right)=\mathbf{x}_{1}-\mathbf{x}_{1}^{\text {ref }}\left(t_{0}\right)$. Substituting this solution into Eq. (III.6), we arrive at the following differential equation for the position operators of the system atoms:

$\mathbf{M}_{11} \ddot{\widetilde{\mathbf{x}}}_{1}(t)=\mathbf{F}_{1}(t)-\mathbf{L}_{11}(t) \widetilde{\mathbf{u}}_{1}(t)+\mathbf{R}_{1}(t)-\int_{t_{0}}^{t} \mathbf{V}_{12}(t) \boldsymbol{\Pi}_{22}(t-\tau)\left[\dot{\mathbf{V}}_{21}(\tau) \widetilde{\mathbf{u}}_{1}(\tau)+\mathbf{V}_{21}(\tau) \dot{\tilde{\mathbf{x}}}_{1}(\tau)\right] d \tau$

where

$$
\begin{aligned}
& \mathbf{F}_{1}(t)=-\mathbf{h}_{1}(t)+\mathbf{V}_{12}(t) \mathbf{D}_{22}^{-1} \mathbf{V}_{2}(t) \\
& \mathbf{L}_{11}(t)=\boldsymbol{\Phi}_{11}(t)-\mathbf{V}_{12}(t) \mathbf{D}_{22}^{-1} \mathbf{V}_{21}(t)
\end{aligned}
$$

are the generalised force and the force-constant matrix for atoms in the open system, respectively. The second terms in the right hand sides of expressions (III.12) and (III.13) are related to the contribution of the vibrating baths atoms. Finally,

$$
\mathbf{R}_{1}(t)=-\mathbf{V}_{12}(t)\left[\dot{\Omega}_{22}\left(t-t_{0}\right) \mathbf{x}_{2}+\Omega_{22}\left(t-t_{0}\right) \mathbf{p}_{2}+\Pi_{22}\left(t-t_{0}\right) \mathbf{g}_{2}\right]
$$

is an operator acting in the Hilbert space of the open system (due to $\mathbf{g}_{2}$, Eq. (III.10)) and of the bath (due to $\mathbf{x}_{2}$ and $\mathbf{p}_{2}$ ). The meaning of this operator will be clarified later on.

In order to solve Eq. (III.11), we need to close this equation with respect to the unknown functions $\mathbf{x}_{1}^{\text {ref }}(t)$. The most obvious way to proceed is to choose these functions to be the instantaneous positions $\left\langle\mathbf{x}_{1}\right\rangle_{t}$ of atoms at time $t$, which are precisely the quantum averages of the position operators $\widetilde{\mathbf{x}}_{1}(t)$ whose dynamical equation has just been derived. In the next section this will enable us to establish a closed set of equations of motion for the atomic positions $\left\langle\mathbf{x}_{1}\right\rangle_{t}$.

In other word, we substitute $x_{i}^{\text {ref }}$ by $\left\langle x_{i}\right\rangle_{t}$ in Eq. (III.11). Now the terms in Eq. (III.11) depend explicitly on the mean values $\left\langle\mathbf{x}_{1}\right\rangle_{t}$ of the atomic positions in the open system (they are not operators). Equation (III.11) is now closed, in the sense that it depends only on the position operators $\widetilde{\mathbf{x}}_{1}(t)$, their time derivatives $\dot{\widetilde{\mathbf{x}}}_{1}(t), \ddot{\widetilde{\mathbf{x}}}_{1}(t)$ and their quantum averages $\left\langle\mathbf{x}_{1}\right\rangle_{t}$. 
It is important to realise that the harmonisation approximation goes beyond the usual harmonic approximation in which the interactions in the Hamiltonian are expanded with respect to fixed atomic positions. The difference is illustarted in Fig. 1 for the interaction $W\left(\mathbf{x}_{1}\right)$. As the time evolves, the expectation value of the atomic positions changes and the expansion of the Hamiltonian changes with them. One may expect that the approximation we made (the second order expansion of the Hamiltonian) is appropriate if fluctuations of atomic positions around the exact expectation values $\left\langle\mathbf{x}_{1}\right\rangle_{t}$ are small with respect to average interactomic distances. This condition is generally true for atoms at standard pressure and temperature, with the possible exception of hydrogen.

Note that formally equations (III.11)-(III.14) correspond to the initial Hamiltonian (II.1)-(II.3): even though the harmonisation approximation was used, the parameters of the Hamiltonian, Eqs. (III.3) and (III.5), depend on time according to the actual system dynamics and the shape of the potential energy terms of the original Hamiltonian.

The obtained equation of motion (III.11) represents what is sometimes called the quantum GLE and which has been known (for somewhat simpler Hamiltonians) since the pioneering work of FKM [21,32]. The main problem associated with this equation is that it is written for operators acting in the whole Hilbert space of the open system and bath. Hence, determination of the expectation values of the atomic positions, the quantities which represent the actual interest, is an additional and rather complex task. Instead, our objective here is to derive an equation directly for these expectation values, the so-called $c$-number GLE for the coordinates of the atoms of the open system, which would enable one to compute average atomic trajectories $\left\langle\mathbf{x}_{1}\right\rangle_{t}$ as a function of time taking full account of the bath. To achieve this goal, we have to multiply both sides of Eq. (III.11) by the initial density matrix $\rho^{0}$ and then take the trace over the whole Hilbert space (system + bath). To accomplish this, we need an explcit expression for the initial density matrix first.

\section{IV. $c$-NUMBER QUANTUM GLE}

We shall start by finding eigenvectors of the bath Hamiltonian (III.5) at the initial time $t_{0}$ in which the displacements of atoms of the system, $\widetilde{\mathbf{u}}_{1}\left(t_{0}\right)=\mathbf{u}_{1}$, are considered as parameters. The Hamiltonian

$$
\mathcal{H}_{b}^{0}=\left(\mathcal{H}_{2}+\mathcal{H}_{12}\right)_{t_{0}}=\frac{1}{2} \mathbf{p}_{2}^{T} \mathbf{p}_{2}+\frac{1}{2} \mathbf{x}_{2}^{T} \mathbf{D}_{22} \mathbf{x}_{2}+\mathbf{g}_{2}^{T} \mathbf{x}_{2}
$$


corresponds to a set of displaced harmonic oscillators (the bath in the presence of the open system) and can be diagonalised exactly using the canonical transformation:

$$
\mathcal{U}^{\dagger} \mathcal{H}_{b}^{0} \mathcal{U}=\sum_{\lambda} \hbar \omega_{\lambda}\left(b_{\lambda}^{\dagger} b_{\lambda}+\frac{1}{2}\right)+E_{\text {pol }}
$$

with $\mathcal{U}=\prod_{\lambda} \mathcal{U}_{\lambda}=\prod_{\lambda} \exp \left(\gamma_{\lambda} b_{\lambda}^{\dagger}-\gamma_{\lambda} b_{\lambda}\right)$ and $E_{\text {pol }}=-\sum_{\lambda} g_{\lambda}^{2} / 2 \omega_{\lambda}^{2}$, where the constants $\gamma_{\lambda}=$ $-g_{\lambda} / \sqrt{2 \hbar} \omega_{\lambda}^{3 / 2}$ with $g_{\lambda}=\mathbf{e}_{\lambda}^{T} \mathbf{g}_{2}$ were introduced. Here $b_{\lambda}^{\dagger}$ and $b_{\lambda}$ are phonon creation and annihilation operators for the mode $\lambda$ satisfying usual commutation relations for bosons. Therefore, the eigenvectors and eigenvalues of $\mathcal{H}_{b}^{0}$ are, respectively: $\left|\psi_{n}\right\rangle=\mathcal{U}|n\rangle=\prod_{\lambda} \mathcal{U}_{\lambda}\left|n_{\lambda}\right\rangle$ and $E_{n}=E_{n}^{0}+E_{p o l}$, where $E_{n}^{0}=\sum_{\lambda} \hbar \omega_{\lambda}\left(n_{\lambda}+\frac{1}{2}\right)$, and

$$
|n\rangle=\prod_{\lambda}\left|n_{\lambda}\right\rangle=\prod_{\lambda} \frac{\left(b_{\lambda}^{\dagger}\right)^{n_{\lambda}}}{\sqrt{n_{\lambda} !}}|0\rangle
$$

is a product of the eigenstates $\left|n_{\lambda}\right\rangle$ of the undisplaced $\lambda$-oscillators, $n=\left\{n_{\lambda}\right\}$ is a set of integer numbers $n_{\lambda}=0,1,2, \ldots$ characterising excitations of each of the oscillators. The states $|n\rangle$ are orthonormal, $\langle n \mid m\rangle=\delta_{n m}$. It is essential to realise that the eigenstates $\left|\psi_{n}\right\rangle$ depend parametrically on the displacements $\mathbf{u}_{1}$ of atoms in the open system (via $\mathbf{g}_{2}$, Eq. (III.10)).

The initial density matrix $\rho^{0}$ of the whole system, corresponding to the bath being at equilibrium with the inverse temperature $\beta=1 / k_{B} T$, can be generally written via eigenstates of the Hamiltonian $\mathcal{H}_{b}^{0}$ as follows:

$$
\rho^{0}=\sum_{n} \rho_{n}^{e q}\left|\psi_{n}\right\rangle \rho_{1}^{0}\left\langle\psi_{n}\right|
$$

where $\rho_{n}^{e q}=\frac{1}{Z_{2}^{0}} e^{-\beta E_{n}^{0}}$ is an eigenvalue of the equilibrium density matrix $\rho_{2}^{e q}=\frac{1}{Z_{2}^{0}} e^{-\beta \mathcal{H}_{2}}$ of (an isolated) bath and $Z_{2}^{0}=\sum_{n} e^{-\beta E_{n}^{0}}$ the corresponding partition function.

Tracing out the states of the bath should reduce this density matrix to the density matrix $\rho_{1}^{0}$ of the open system at the initial time, and with the choice made above, this is indeed the case:

$$
\operatorname{Tr}_{2}\left(\rho^{0}\right)=\sum_{m}\left\langle\psi_{m}\left|\rho^{0}\right| \psi_{m}\right\rangle=\sum_{m}\left\langle\psi_{m}\left|\left\{\sum_{n}\left|\psi_{n}\right\rangle \rho_{n}^{e q} \rho_{1}^{0}\left\langle\psi_{n}\right|\right\}\right| \psi_{m}\right\rangle=\left(\sum_{m} \rho_{m}^{e q}\right) \rho_{1}^{0}=\rho_{1}^{0}
$$

When calculating the trace over the bath, we used the eigenstates $\left|\psi_{n}\right\rangle$ of $\mathcal{H}_{b}^{0}$. Note also that $\sum_{m} \rho_{m}^{e q}=1$ due to normalisation of the bath density matrix $\rho_{2}^{e q}$.

Note that this expression is partition-free, i.e. it is not based on the usually invoked Born approximation $[1,39]$. This is because the initial density matrix $\rho_{1}^{0}$ of the open system depends on the atomic positions there and hence cannot be taken outside the sum over states $\left|\psi_{n}\right\rangle$ which also depend explicitly on these positions (via $\mathbf{g}_{2}$ ). 
In order to introduce a stochastic field into our formulation, we realise that generally any density matrix can always be expanded in terms of the eigenstates of the bath: $\rho=\sum_{n m}\left|\psi_{n}\right\rangle \rho_{n m}^{\prime}\left\langle\psi_{m}\right|$, where $\rho_{n m}^{\prime}$ are operators acting within the Hilbert space of the open system. This expression is exact and also partition-free. Inspired by the work of Ref. [54], we choose the operator-coefficients $\rho_{n m}^{\prime}$ in the initial density matrix as

$$
\rho_{n m}^{\prime}=\rho_{n m} e^{i\left(\theta_{n}-\theta_{m}\right)}=\rho_{n m} \prod_{\lambda} e^{i\left(\theta_{n_{\lambda}}-\theta_{m_{\lambda}}\right)}
$$

where $\theta_{n_{\lambda}}$ are random numbers (phases) uniformly distributed between 0 and $2 \pi$ and $\rho_{n m}$ are operators acting in the Hilbert space of the open system only. Hence the following ansatz is proposed for the initial density matrix of the whole system:

$$
\rho^{S}=\sum_{n m} e^{i\left(\theta_{n}-\theta_{m}\right)}\left|\psi_{n}\right\rangle \rho_{n m}\left\langle\psi_{m}\right|
$$

The density matrix written in this way can be thought of as being expanded in terms of the bath exact oscillatory functions, $\left|\psi_{n}\right\rangle e^{i \theta_{n}}$, which contain random phases $\theta_{n}$. We have indicated explicitly with the superscript $S$ that this density matrix is stochastic in nature.

Averages with respect to the random field of phases will be denoted with the over-bar. Importantly,

$$
\overline{e^{i \theta_{n}}}=\prod_{\lambda} \overline{e^{i \theta_{\lambda}}}=\prod_{\lambda} \frac{1}{2 \pi} \int_{0}^{2 \pi} e^{i \theta_{n_{\lambda}}} d \theta_{n_{\lambda}}=0
$$

so that, when $n \neq m$ (i.e. $n_{\lambda} \neq m_{\lambda}$ for at least one mode $\lambda$ )

$$
\overline{e^{i\left(\theta_{n}-\theta_{m}\right)}}=\left(\prod_{\lambda \in\left\{n_{\lambda} \neq m_{\lambda}\right\}} \overline{e^{i \theta_{n}} e^{-i \theta_{m_{\lambda}}}}\right)\left(\prod_{\lambda \in\left\{n_{\lambda}=m_{\lambda}\right\}} \overline{1}\right)=\prod_{\lambda \in\left\{n_{\lambda} \neq m_{\lambda}\right\}} \overline{e^{i \theta_{n_{\lambda}}}}{\overline{e^{i \theta_{m_{\lambda}}}}}^{*}=0
$$

while if $n=m$ (i.e. $n_{\lambda}=m_{\lambda}$ for any $\lambda$ ) we have $\left[\overline{e^{i\left(\theta_{n}-\theta_{m}\right)}}\right]_{n=m}=\overline{1}=\prod_{\lambda} \frac{1}{2 \pi} \int_{0}^{2 \pi} d \theta_{n_{\lambda}}=1$. Hence, generally

$$
\overline{e^{i\left(\theta_{n}-\theta_{m}\right)}}=\prod_{\lambda} \delta_{n_{\lambda} m_{\lambda}}=\delta_{n m}
$$

To introduce the temperature into our description, we postulate that the stochastic field average of $\rho^{S}$,

$$
\overline{\rho^{S}}=\sum_{n m} \overline{e^{i\left(\theta_{n}-\theta_{m}\right)}}\left|\psi_{n}\right\rangle \rho_{n m}\left\langle\psi_{m}\left|=\sum_{n}\right| \psi_{n}\right\rangle \rho_{n n}\left\langle\psi_{n}\right|
$$

coincides with the exact initial density matrix of Eq. (IV.2): $\overline{\rho^{S}} \equiv \rho^{0}$. This procedure sets up only diagonal elements of the operators as $\rho_{n n}=\rho_{1}^{0} \rho_{n}^{e q}$; non-diagonal operators $\rho_{n m}$ still remain undetermined at this stage. Note that $\operatorname{Tr}_{2}\left(\rho^{S}\right)=\sum_{n} \rho_{n n}=\rho_{1}^{0}$. 
To obtain an equation for the exact averages $\left\langle\mathbf{x}_{1}\right\rangle_{t}^{S}$ for atoms in the open system, we multiply both sides of Eq. (III.11) by $\rho^{S}$ from (IV.4) and then take the trace over the whole Hilbert space (using eigenstates of $\left.\mathcal{H}_{b}^{0}\right)$. The superscript $S$ in $\left\langle\mathbf{x}_{1}\right\rangle_{t}^{S}=\operatorname{Tr}\left(\rho^{S} \widetilde{\mathbf{x}}_{1}(t)\right)$ indicates that a particular manifestation of the stochastic field (a particular set of random phases) is used. Note that, when calculating a given trajectory, the harmonisation approximation is made with respect to these particular averages corresponding to the given realisation of the stochastic field. Then, multiplying both sides of Eq. (III.11) by $\rho^{S}$, taking the trace and noting that $\operatorname{Tr}\left(\rho^{S} \widetilde{\mathbf{u}}_{1}(t)\right)=$ $\operatorname{Tr}\left(\rho^{S} \widetilde{\mathbf{x}}_{1}(t)\right)-\left\langle\mathbf{x}_{1}\right\rangle_{t}^{S}=0$ for any time, we obtain the desired cQGLE:

$$
\mathbf{M}_{11} \partial_{t}^{2}\left\langle\mathbf{x}_{1}\right\rangle_{t}^{S}=\mathbf{F}_{1}(t)+\boldsymbol{R}_{1}(t)-\int_{t_{0}}^{t} \mathbf{K}_{11}(t, \tau) \partial_{t}\left\langle\mathbf{x}_{1}\right\rangle_{\tau}^{S} d \tau
$$

where $\mathbf{K}_{11}(t, \tau)$ is the friction kernel (II.13), $\mathbf{F}_{1}(t)$ is the conservative force (III.12) containing a "polaron"-like contribution from the bath (cf. [17, 18]) elastically responding to the atomic positions in the open system and

$$
\boldsymbol{\mathcal { R }}_{1}(t)=\operatorname{Tr}\left(\rho^{S} \mathbf{R}_{1}(t)\right)=\operatorname{Tr}_{1}\left\langle\mathbf{R}_{1}(t)\right\rangle_{2}^{S}
$$

is the random force, where $\langle\ldots\rangle_{2}^{S}=\operatorname{Tr}_{2}\left(\rho^{S} \ldots\right)$ is the statistical average over the bath, while $\operatorname{Tr}_{1}$ corresponds to the trace over the states of the open system.

Importantly, the derived cQGLE is self-contained as all of the time-dependent terms in it are explicit functions of the averages $\left\langle\mathbf{x}_{1}\right\rangle_{t}^{S}$ which this equation defines. At first site, the cQGLE has the same form as the classical GLE $[17,18]$. However, the behaviour of the random force, as will be shown below, is very different from the classical case.

\section{PROPERTIES OF THE RANDOM FORCE}

The random force (IV.8) contains the random phases associated with all harmonic oscillators of the bath; at the same time, it depends explcitily on time and hence represents a stochastic process. To define explicitly the cQGLE, it is necessary to study this stochastic process in more detail. In particular, we would like to establish whether the random force $\boldsymbol{\mathcal { R }}_{1}(t)=\left(\mathcal{R}_{i}(t) ; i \in 1\right)$ is a Gaussian or a non-Gaussian process. It is known [55] that if the process is Gaussian, then odd-moment correlation functions must be equal to zero, while even-moment correlation functions must be equal to a sum of products of all pair correlation functions. We shall explicitly show here that if the first statement appears to be true, the second one is not, proving that the stochastic field, which we have introduced above, in not Gaussian. 


\section{A. Random force}

To calculate the random force, we first take the trace of the operator (II.14) over the bath states:

$$
\left\langle\mathbf{R}_{1}(t)\right\rangle_{2}^{S}=-\mathbf{V}_{12}(t)\left[\dot{\Omega}_{22}\left(t-t_{0}\right)\left\langle\mathbf{x}_{2}\right\rangle_{2}^{S}+\mathbf{\Omega}_{22}\left(t-t_{0}\right)\left\langle\mathbf{p}_{2}\right\rangle_{2}^{S}+\boldsymbol{\Pi}_{22}\left(t-t_{0}\right)\left\langle\mathbf{g}_{2}\right\rangle_{2}^{S}\right]
$$

where

$$
\begin{gathered}
\left\langle\mathbf{x}_{2}\right\rangle_{2}^{S}=\sum_{m n}\left\langle\psi_{m}\left|\mathbf{x}_{2}\right| \psi_{n}\right\rangle \rho_{n m} e^{i\left(\theta_{n}-\theta_{m}\right)} \\
\left\langle\mathbf{p}_{2}\right\rangle_{2}^{S}=\sum_{m n}\left\langle\psi_{m}\left|\mathbf{p}_{2}\right| \psi_{n}\right\rangle \rho_{n m} e^{i\left(\theta_{n}-\theta_{m}\right)} \\
\left\langle\mathbf{g}_{2}\right\rangle_{2}^{S}=\left\langle\mathbf{V}_{2}\left(t_{0}\right)+\mathbf{V}_{21}\left(t_{0}\right) \mathbf{u}_{1}\right\rangle_{2}^{S}=\rho_{1}^{0}\left(\mathbf{V}_{2}\left(t_{0}\right)+\mathbf{V}_{21}\left(t_{0}\right) \mathbf{u}_{1}\right)=\rho_{1}^{0} \mathbf{g}_{2}
\end{gathered}
$$

The matrix elements $\left\langle\psi_{m}\left|\mathbf{x}_{2}\right| \psi_{n}\right\rangle$ and $\left\langle\psi_{m}\left|\mathbf{p}_{2}\right| \psi_{n}\right\rangle$ which are needed for calculating the averages $\left\langle\mathbf{x}_{2}\right\rangle_{2}$ and $\left\langle\mathbf{p}_{2}\right\rangle_{2}$, are obtained by making use of the explicit expressions for the position and momenta operators (written via bath modes creation and annihilation operators),

$$
\begin{aligned}
& \mathbf{x}_{2}=\sum_{\lambda} \sqrt{\frac{\hbar}{2 \omega_{\lambda}}} \mathbf{e}_{\lambda}\left(b_{\lambda}^{\dagger}+b_{\lambda}\right), \\
& \mathbf{p}_{2}=i \sum_{\lambda} \sqrt{\frac{\hbar \omega_{\lambda}}{2}} \mathbf{e}_{\lambda}\left(b_{\lambda}^{\dagger}-b_{\lambda}\right),
\end{aligned}
$$

the fact that $\left|\psi_{n}\right\rangle=\mathcal{U}|n\rangle$, and also that $\mathcal{U}^{\dagger} b_{\lambda} \mathcal{U}=b_{\lambda}+\gamma_{\lambda}$ and $\mathcal{U}^{\dagger} b_{\lambda}^{\dagger} \mathcal{U}=b_{\lambda}^{\dagger}+\gamma_{\lambda}$. We have:

$$
\begin{gathered}
\left\langle\psi_{m}\left|\mathbf{x}_{2}\right| \psi_{n}\right\rangle=\sum_{\lambda} \sqrt{\frac{\hbar}{2 \omega_{\lambda}}} \mathbf{e}_{\lambda}\left\langle m\left|\mathcal{U}^{\dagger}\left(b_{\lambda}^{\dagger}+b_{\lambda}\right) \mathcal{U}\right| n\right\rangle \\
=\delta_{n m} \sum_{\lambda} \sqrt{\frac{2 \hbar}{\omega_{\lambda}}} \mathbf{e}_{\lambda} \gamma_{\lambda}+\sum_{\lambda} \sqrt{\frac{\hbar}{2 \omega_{\lambda}}} \mathbf{e}_{\lambda}\left\langle m\left|b_{\lambda}^{\dagger}+b_{\lambda}\right| n\right\rangle \\
=-\delta_{m n} \mathbf{D}_{22}^{-1} \mathbf{g}_{2}+\sum_{\lambda} \sqrt{\frac{\hbar}{2 \omega_{\lambda}}} \mathbf{e}_{\lambda}\left\langle m\left|b_{\lambda}^{\dagger}+b_{\lambda}\right| n\right\rangle
\end{gathered}
$$

Note that the matrix element in the second term is zero if $m=n$. Hence, we obtain

$$
\left\langle\mathbf{x}_{2}\right\rangle_{2}^{S}=-\rho_{1}^{0} \mathbf{D}_{22}^{-1} \mathbf{g}_{2}+\sum_{m \neq n} \rho_{n m} e^{i\left(\theta_{n}-\theta_{m}\right)} \sum_{\lambda} \sqrt{\frac{\hbar}{2 \omega_{\lambda}}} \mathbf{e}_{\lambda}\left\langle m\left|b_{\lambda}^{\dagger}+b_{\lambda}\right| n\right\rangle
$$

Similarly, one has:

$$
\left\langle\mathbf{p}_{2}\right\rangle_{2}^{S}=i \sum_{m \neq n} \rho_{n m} e^{i\left(\theta_{n}-\theta_{m}\right)} \sum_{\lambda} \sqrt{\frac{\hbar \omega_{\lambda}}{2}} \mathbf{e}_{\lambda}\left\langle m\left|b_{\lambda}^{\dagger}-b_{\lambda}\right| n\right\rangle
$$


Substituting Eqs. (V.7), (V.8) and (V.4) into Eq. (V.1), and noticing that $\dot{\Omega}_{22}\left(t-t_{0}\right) \mathbf{D}_{22}^{-1}=$ $\Pi_{22}\left(t-t_{0}\right)$ [17], we observe that the only dependence on the states of the open system in $\left\langle\mathbf{R}_{1}(t)\right\rangle_{2}$ comes from the operators $\rho_{m n}$. Therefore, taking the trace over the states of the open system, one obtains the following equation for the component $i \in 1$ of the random force:

$$
\mathcal{R}_{i}(t)=\sum_{m \neq n} e^{i\left(\theta_{n}-\theta_{m}\right)} Z_{i}^{m n}(t)
$$

where the coefficients

$$
Z_{i}^{m n}(t)=A_{m n} \sum_{\lambda}\left[D_{i \lambda}(t)\left\langle m\left|b_{\lambda}^{\dagger}\right| n\right\rangle+D_{i \lambda}^{*}(t)\left\langle m\left|b_{\lambda}\right| n\right\rangle\right]
$$

do not contain the random phases. Here

$$
D_{i \lambda}(t)=-\sqrt{\frac{\hbar}{2 \omega_{\lambda}}} V_{i \lambda}(t) e^{i \omega_{\lambda}\left(t-t_{0}\right)}
$$

and

$$
V_{i \lambda}(t)=\sum_{j \in 2} V_{i j}(t) e_{\lambda j}
$$

and we have used Eqs. (II.8) and (II.9) for the matrices $\Omega_{22}$ and $\dot{\Omega}_{22}$. It is easy to see that $Z_{i}^{m n}(t)^{*}=Z_{i}^{n m}(t)$. The numbers

$$
A_{m n}=\operatorname{Tr}_{1}\left(\rho_{m n}\right)
$$

depend on the unknown operators $\rho_{m n}$ which act in the Hilbert space of the open system and correspond to the initial time $t_{0}$. Hence, in principle $A_{m n}$ would depend on the initial preparation of the open system.

Recall that $n$ and $m$ here represent sets of positive integers (including zero) corresponding to quantum numbers of all vibrational modes, i.e. $n=\left\{n_{\lambda}\right\}$ and $m=\left\{m_{\lambda}\right\}$. Two such sets are considered different if at least for one mode $\lambda_{1}$ the quantum numbers differ, i.e. $n_{\lambda_{1}} \neq m_{\lambda_{1}}$. In the following, to simplify the notations, it is convenient to "forget" that $n$ and $m$ are the sets of numbers and assume that they are simply integer numbers themselves.

Before considering the correlation functions, we note that the average of the random force, $\overline{\mathcal{R}_{i}(t)}$, is zero since according to Eq. (IV.5) the double sum in Eq. (V.9) does not contain the term $m=n$. Hence, the first moment of the random process is zero. 
(c)

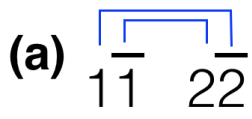

(b)

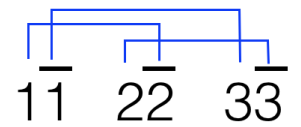

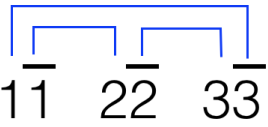

(1)
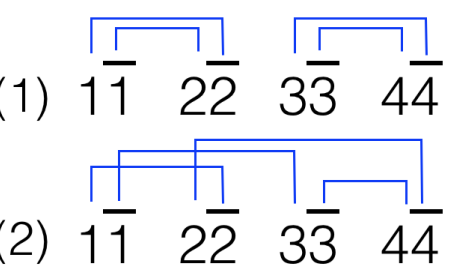

(3)

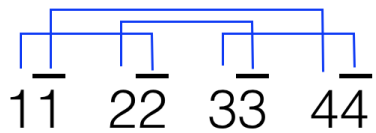

(4) $1 \overline{1} \quad 2 \overline{2} \quad 3 \overline{3} \quad 4 \overline{4}$

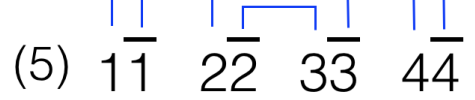

(6)

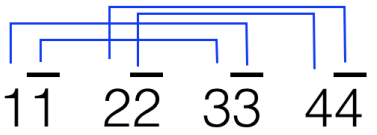

(7) $1 \frac{\sqrt{1}}{2} \frac{\sqrt{2}}{3} \quad \frac{\sqrt{3}}{4} \quad \frac{1}{4}$

(8) $\begin{array}{llll}1 \overline{1} & 2 \overline{2} & 3 \overline{3} & 4 \overline{4}\end{array}$

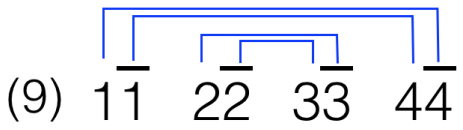

Figure 2. Schematic representation of all non-zero pairings of phases for second (a), third (b) and fourth (c) order correlation functions. Numbers 1, 2, etc. correspond to states $n_{1}, n_{2}$, etc. (they have the plus sign in the exponential factors), while a number with the bar on top, $\overline{1}, \overline{2}$, etc. (they have the minus sign in the exponentials) are associated with the states $m_{1}, m_{2}$, and so on. The pairing method is somewhat similar to the well-known Wick's theorem of the many-body quantum statistical mechanics if numbers without the bar on top of them are associated with annihilation operators, while the ones with the bar with creation operators; the only difference is that pairing of the same numbers is forbidden in our case.

\section{B. Pair correlation function}

We next calculate the second order correlation function:

$$
\overline{\mathcal{R}_{i_{1}}\left(t_{1}\right) \mathcal{R}_{i_{2}}\left(t_{2}\right)}=\sum_{m_{1} \neq n_{1}} \sum_{m_{2} \neq n_{2}} \overline{e^{i\left(\theta_{n_{1}}-\theta_{m_{1}}\right)} e^{i\left(\theta_{n_{2}}-\theta_{m_{2}}\right)}} Z_{i_{1}}^{m_{1} n_{1}}\left(t_{1}\right) Z_{i_{2}}^{m_{2} n_{2}}\left(t_{2}\right)
$$

When taking the average of the exponential factors with the phases, the integers $n_{j}$ and $m_{j}$ $(j=1,2)$ may take all possible values; they can all be different or equal, subject to the condition that $n_{j} \neq m_{j}$ for any $j=1,2$ (see Eq. (V.9)). Because of this condition, three or four integers cannot be equal; a nonzero value of the average of the exponentials in the above expression can only be possible if the four integers split into two pairs of equal integers. Since $n_{1} \neq m_{1}$ and $n_{2} \neq m_{2}$, only two possibilities remain which are: (i) $n_{1}=n_{2}, m_{1}=m_{2}$ and (ii) $n_{1}=m_{2}, m_{1}=n_{2}$. It is easy to see that in the former case the contribution is zero:

$\overline{e^{i\left(\theta_{n_{1}}-\theta_{m_{1}}\right)} e^{i\left(\theta_{n_{2}}-\theta_{m_{2}}\right)}}=\overline{e^{i\left(\theta_{n_{1}}-\theta_{m_{1}}\right)} e^{i\left(\theta_{n_{1}}-\theta_{m_{1}}\right)}}=\overline{e^{2 i \theta_{n_{1}}} e^{-2 i \theta_{m_{1}}}}=\overline{e^{2 i \theta_{n_{1}}}} \overline{e^{-2 i \theta_{m_{1}}}}=\overline{e^{2 i \theta_{n_{1}}}}\left(\overline{e^{2 i \theta_{m_{1}}}}\right)^{*}=0$

However, in the second case we obtain a nonzero result,

$$
\overline{e^{i\left(\theta_{n_{1}}-\theta_{m_{1}}\right)} e^{i\left(\theta_{n_{2}}-\theta_{m_{2}}\right)}}=\overline{e^{i\left(\theta_{n_{1}}-\theta_{m_{1}}\right)} e^{i\left(\theta_{m_{1}}-\theta_{n_{1}}\right)}}=\overline{e^{i\left(\theta_{n_{1}}-\theta_{n_{1}}\right)} e^{-i\left(\theta_{m_{1}}-\theta_{m_{1}}\right)}}=\overline{1} \overline{1}=1
$$




$$
\begin{array}{ll}
\text { (a) } \overline{n_{1}} & A_{n_{1} n_{2}} D_{i \lambda}^{*}(t)\left\langle n_{1}\left|b_{\lambda}\right| n_{2}\right\rangle \\
\text { (b) } \overline{n_{1}}: \frac{}{n_{2}} & A_{n_{1} n_{2}} D_{i \lambda}(t)\left\langle n_{1}\left|b_{\lambda}^{\dagger}\right| n_{2}\right\rangle
\end{array}
$$

Figure 3. Two elementary graphs and the corresponding expressions associated with them: (a) step "up" and (b) step "down". In either case the integer numbers $n_{1}=\left\{n_{1 \lambda^{\prime}}\right\}$ and $n_{2}=\left\{n_{2 \lambda^{\prime}}\right\}$ satisfy the conditions: $n_{1 \lambda^{\prime}}=n_{2 \lambda^{\prime}}$ for any $\lambda^{\prime} \neq \lambda$, and $n_{1 \lambda}=n_{2 \lambda} \pm 1$ otherwise.

yielding

$$
\overline{\mathcal{R}_{i_{1}}\left(t_{1}\right) \mathcal{R}_{i_{2}}\left(t_{2}\right)}=\sum_{m_{1} \neq n_{1}} \sum_{m_{2} \neq n_{2}} \delta_{n_{1} m_{2}} \delta_{m_{1} n_{2}} Z_{i_{1}}^{m_{1} n_{1}}\left(t_{1}\right) Z_{i_{2}}^{m_{2} n_{2}}\left(t_{2}\right)=\sum_{n_{1} n_{2}} Z_{i_{1}}^{n_{2} n_{1}}\left(t_{1}\right) Z_{i_{2}}^{n_{1} n_{2}}\left(t_{2}\right)
$$

We conclude, that when pairing phases during averaging, the only non-zero contribution came by pairing phases which have opposite signs in the exponentials (the second case). This particular pairing can be associated with a simple diagram shown in Fig. 2(a).

Next, substituting into the last expression the explicit formula (V.10) for the $Z$-coefficients, we obtain:

$$
\begin{aligned}
\overline{\mathcal{R}_{i_{1}}\left(t_{1}\right) \mathcal{R}_{i_{2}}\left(t_{2}\right)}= & \sum_{n_{1} n_{2}} A_{n_{2} n_{1}} A_{n_{1} n_{2}} \sum_{\lambda_{1} \lambda_{2}}\left[D_{i_{1} \lambda_{1}}\left\langle n_{2}\left|b_{\lambda_{1}}^{\dagger}\right| n_{1}\right\rangle+D_{i_{1} \lambda_{1}}^{*}\left\langle n_{2}\left|b_{\lambda_{1}}\right| n_{1}\right\rangle\right] \\
& \times\left[D_{i_{2} \lambda_{2}}\left\langle n_{1}\left|b_{\lambda_{2}}^{\dagger}\right| n_{2}\right\rangle+D_{i_{2} \lambda_{2}}^{*}\left\langle n_{1}\left|b_{\lambda_{2}}\right| n_{2}\right\rangle\right]
\end{aligned}
$$

where the time arguments have been omitted (they can easily be restored: the time $t_{j}$ is placed according to $\left.D_{i_{j} \lambda_{j}} \rightarrow D_{i_{j} \lambda_{j}}\left(t_{j}\right)\right)$. It is clear that after opening the square brackets there will be four terms containing the following product of matrix elements:

$$
\left\langle n_{2}\left|b_{\lambda_{1}}^{(\dagger)}\right| n_{1}\right\rangle\left\langle n_{1}\left|b_{\lambda_{2}}^{(\dagger)}\right| n_{2}\right\rangle
$$

where the dagger inside the round brackets means that the dagger either might be there or not. Here the phonon modes $\lambda_{1}$ and $\lambda_{2}$ are independent. Clearly, no matter whether the daggers are present or not, this product of the matrix elements can only be non-zero if $\lambda_{1}=\lambda_{2}$. Then, we need to consider four expressions which contain

$$
\left\langle n_{2}\left|b_{\lambda}^{(\dagger)}\right| n_{1}\right\rangle\left\langle n_{1}\left|b_{\lambda}^{(\dagger)}\right| n_{2}\right\rangle
$$

with the same phonon index $\lambda$. Depending on the particular combination of daggers in the above expression, it can be either zero or non-zero. It is convenient to associate a simple graph with each 

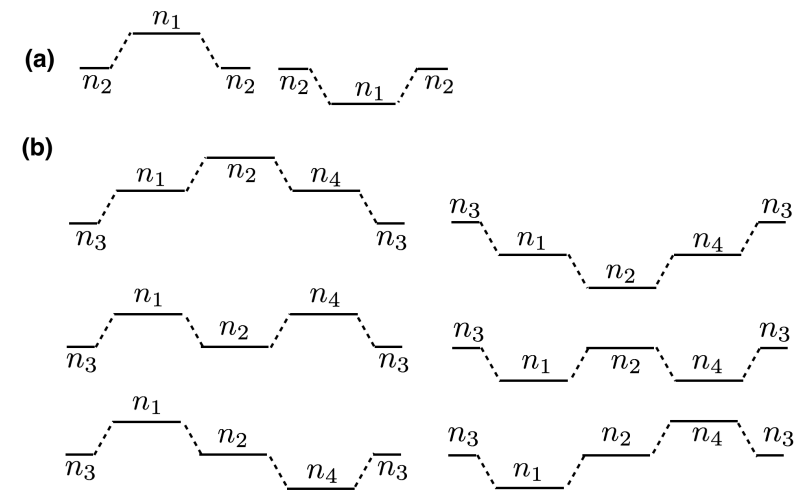

Figure 4. All step-graphs which lead to non-zero contributions for the second order (a) and fourth order (b) correlation functions.

term in the $Z$-coefficient (V.10), see Fig. 3. A non-zero contribution appears if the appropriate combination of two elementary graphs like those shown starts and ends at the same state $n_{2}$, see Fig. 4(a). If the left graph in Fig. 4(a) results in the contribution

$$
\begin{aligned}
& A_{n_{2} n_{1}} D_{i_{1} \lambda}^{*}\left(t_{1}\right)\left\langle n_{2}\left|b_{\lambda}\right| n_{1}\right\rangle A_{n_{1} n_{2}} D_{i_{2} \lambda}\left(t_{2}\right)\left\langle n_{1}\left|b_{\lambda}^{\dagger}\right| n_{2}\right\rangle \\
= & \frac{\hbar}{2 \omega_{\lambda}}\left|A_{n_{1} n_{2}}\left\langle n_{1}\left|b_{\lambda}^{\dagger}\right| n_{2}\right\rangle\right|^{2} V_{i_{1} \lambda}\left(t_{1}\right) V_{i_{2} \lambda}\left(t_{2}\right) e^{-i \omega_{\lambda}\left(t_{1}-t_{2}\right)}
\end{aligned}
$$

then the right one yields

$$
\begin{aligned}
& A_{n_{2} n_{1}} D_{i_{1} \lambda}\left(t_{1}\right)\left\langle n_{2}\left|b_{\lambda}^{\dagger}\right| n_{1}\right\rangle A_{n_{1} n_{2}} D_{i_{2} \lambda}^{*}\left(t_{2}\right)\left\langle n_{1}\left|b_{\lambda}\right| n_{2}\right\rangle \\
= & \frac{\hbar}{2 \omega_{\lambda}}\left|A_{n_{1} n_{2}}\left\langle n_{2}\left|b_{\lambda}^{\dagger}\right| n_{1}\right\rangle\right|^{2} V_{i_{1} \lambda}\left(t_{1}\right) V_{i_{2} \lambda}\left(t_{2}\right) e^{i \omega_{\lambda}\left(t_{1}-t_{2}\right)}
\end{aligned}
$$

Summing up both terms and changing the summation indices $n_{1} \longleftrightarrow n_{2}$ in the second term, we arrive at a very simple result:

$$
\overline{\mathcal{R}_{1}(t) \boldsymbol{\mathcal { R }}_{1}^{T}\left(t^{\prime}\right)}=\mathbf{V}_{12}(t)\left[\sum_{\lambda} A_{\lambda} \mathbf{e}_{\lambda} \mathbf{e}_{\lambda}^{T} \cos \left(\omega_{\lambda}\left(t-t^{\prime}\right)\right)\right] \mathbf{V}_{21}\left(t^{\prime}\right)
$$

By taking the transpose of both sides, it is seen that this autocorrelation function is symmetric with respect to the permutation of times:

$$
\left[\overline{\mathcal{R}_{1}(t) \mathcal{R}_{1}^{T}\left(t^{\prime}\right)}\right]^{T}=\overline{\mathcal{R}_{1}\left(t^{\prime}\right) \mathcal{R}_{1}^{T}(t)}
$$

Nothing can be said at this stage about the amplitudes

$$
A_{\lambda}=\frac{\hbar}{\omega_{\lambda}} \sum_{m \neq n}\left|A_{n m}\right|^{2}\left|\left\langle m\left|b_{\lambda}\right| n\right\rangle\right|^{2}
$$


apart from the fact that these must depend on the temperature and the initial preparation of the system. Note also that here we basically have a single summation over phonon states of the bath since

$$
\left\langle m\left|b_{\lambda}\right| n\right\rangle=\left\langle\left\{m_{\lambda^{\prime}}\right\}\left|b_{\lambda}\right|\left\{n_{\lambda^{\prime}}\right\}\right\rangle=\sqrt{n_{\lambda}} \delta_{n_{\lambda}-1, m_{\lambda}}\left(\prod_{\lambda^{\prime} \neq \lambda} \delta_{n_{\lambda^{\prime}}, m_{\lambda^{\prime}}}\right)
$$

Surprisingly, the pair correlation function (V.15) has the same structure as the one in the classical GLE $[17,18]$ derived for the same Hamiltonian $\mathcal{H}$, where $A_{\lambda}=1 / \beta \omega_{\lambda}^{2}$. Therefore, the unknown amplitudes $A_{\lambda}$ in Eq. (V.15) are expected to tend to this limit as $\hbar, \beta \rightarrow 0$.

\section{Odd order correlation functions}

We next consider the third order correlation function

$$
\begin{gathered}
\overline{\mathcal{R}_{i_{1}}\left(t_{1}\right) \mathcal{R}_{i_{2}}\left(t_{2}\right) \mathcal{R}_{i_{3}}\left(t_{3}\right)} \\
=\sum_{m_{1} \neq n_{1}} \sum_{m_{2} \neq n_{2}} \sum_{m_{3} \neq n_{3}} e^{\overline{e^{i\left(\theta_{n_{1}}-\theta_{m_{1}}\right)}} e^{i\left(\theta_{n_{2}}-\theta_{m_{2}}\right)} e^{i\left(\theta_{n_{3}}-\theta_{m_{3}}\right)}} Z_{i_{1}}^{m_{1} n_{1}}\left(t_{1}\right) Z_{i_{2}}^{m_{2} n_{2}}\left(t_{2}\right) Z_{i_{3}}^{m_{3} n_{3}}\left(t_{3}\right)
\end{gathered}
$$

We ought to analyse the average of the product of the three exponentials with the phases. In the sum above, the numbers $n_{j}$ and $m_{j}(j=1,2,3)$ may all have different values and also there will be identical values. Since there are limitations on the values of the possible integers (recall that $n_{j} \neq m_{j}$ for any $\left.j=1,2,3\right)$, then four, five or six integers cannot be identical. If any three integers are identical, e.g. $n_{1}=n_{2}=m_{3}$, then the other three must form another triple of identical numbers as well, $m_{1}=m_{2}=n_{3}$, since otherwise the expression would contain the average of an exponential of a single phase, $\overline{e^{i \theta}}$, which is zero. So, in this case

$$
\begin{aligned}
& \overline{e^{i\left(\theta_{n_{1}}-\theta_{m_{1}}\right)} e^{i\left(\theta_{n_{2}}-\theta_{m_{2}}\right)} e^{i\left(\theta_{n_{3}}-\theta_{m_{3}}\right)}} \Rightarrow \overline{e^{i\left(\theta_{n_{1}}-\theta_{m_{1}}\right)} e^{i\left(\theta_{n_{1}}-\theta_{m_{1}}\right)} e^{i\left(\theta_{m_{1}}-\theta_{n_{1}}\right)}} \\
& =\overline{e^{i \theta_{n_{1}}} e^{-i \theta_{m_{1}}}}=\overline{e^{i \theta_{n_{1}}}}\left(\overline{e^{i \theta_{m_{1}}}}\right)^{*}=0
\end{aligned}
$$

Hence, the only possibility that remains is the one in which the six integers are split into three pairs of identical integers. We have to pair up the integers of complex conjugate exponentials (i.e. exponentials with plus and minus signs in their exponents) as otherwise we have zero again. Indeed, if, for instance, $n_{1}=n_{2}$, then the total average would contain a factor

$$
\overline{e^{i \theta_{n_{1}}} e^{i \theta_{n_{2}}}} \Rightarrow \overline{e^{i \theta_{n_{1}}} e^{i \theta_{n_{1}}}}=\overline{e^{2 i \theta_{n_{1}}}}=0
$$


Thus, pairing only complex conjugate exponentials, we have two possibilities shown schematically in Fig. 2(b): (i) $n_{1}=m_{2}, m_{1}=n_{3}, n_{2}=m_{3}$ and (ii) $n_{1}=m_{3}, m_{1}=n_{2}, m_{2}=n_{3}$. Therefore, one can write:

$$
\begin{aligned}
& \overline{e^{i\left(\theta_{n_{1}}-\theta_{m_{1}}\right)} e^{i\left(\theta_{n_{2}}-\theta_{m_{2}}\right)} e^{i\left(\theta_{n_{3}}-\theta_{m_{3}}\right)}}= \\
& =\delta_{n_{1} m_{2}} \delta_{m_{1} n_{3}} \delta_{n_{2} m_{3}} \overline{e^{i\left(\theta_{n_{1}}-\theta_{m_{1}}\right)} e^{i\left(\theta_{n_{2}}-\theta_{n_{1}}\right)} e^{i\left(\theta_{m_{1}}-\theta_{n_{2}}\right)}}+\delta_{n_{1} m_{3}} \delta_{m_{1} n_{2}} \delta_{m_{2} n_{3}} e^{i\left(\theta_{n_{1}}-\theta_{m_{1}}\right)} e^{i\left(\theta_{m_{1}}-\theta_{m_{2}}\right)} e^{i\left(\theta_{m_{2}}-\theta_{n_{1}}\right)} \\
& =\delta_{n_{1} m_{2}} \delta_{m_{1} n_{3}} \delta_{n_{2} m_{3}} \overline{1} \overline{1} \overline{1}+\delta_{n_{1} m_{3}} \delta_{m_{1} n_{2}} \delta_{m_{2} n_{3}} \overline{1} \overline{1} \overline{1}=\delta_{n_{1} m_{2}} \delta_{m_{1} n_{3}} \delta_{n_{2} m_{3}}+\delta_{n_{1} m_{3}} \delta_{m_{1} n_{2}} \delta_{m_{2} n_{3}}
\end{aligned}
$$

which results in the correlation function

$\overline{\mathcal{R}_{i_{1}}\left(t_{1}\right) \mathcal{R}_{i_{2}}\left(t_{2}\right) \mathcal{R}_{i_{3}}\left(t_{3}\right)}=\sum_{n_{1}} \sum_{n_{2}} \sum_{n_{3}}\left[Z_{i_{1}}^{n_{3} n_{1}}\left(t_{1}\right) Z_{i_{2}}^{n_{1} n_{2}}\left(t_{2}\right) Z_{i_{3}}^{n_{2} n_{3}}\left(t_{3}\right)+Z_{i_{1}}^{n_{2} n_{1}}\left(t_{1}\right) Z_{i_{2}}^{n_{3} n_{2}}\left(t_{2}\right) Z_{i_{3}}^{n_{1} n_{3}}\left(t_{3}\right)\right]$

We shall show now that either of the two terms is actually equal to zero. As the second term is the complex conjugate of the first, it is sufficient to consider the latter. Using the explicit expression (V.10) for the $Z$-coefficients and multiplying all terms out, we arrive at an expression containing 8 terms altogether, each of them being proportional to a product of three matrix elements:

$$
\left\langle n_{3}\left|b_{\lambda}^{(\dagger)}\right| n_{1}\right\rangle\left\langle n_{1}\left|b_{\lambda}^{(\dagger)}\right| n_{2}\right\rangle\left\langle n_{2}\left|b_{\lambda}^{(\dagger)}\right| n_{3}\right\rangle
$$

where we have already set indices of all the three phonon modes to be the same and equal to $\lambda$, as otherwise the result would be zero. Going from left to right, in this product we start from the state $n_{3}$ and must end at the same state. Clearly, that should require the same number of steps "up" and "down" on the step graphs, which is impossible with the three steps available here. Hence, for any combination of daggers in the above expression the product of the matrix elements is zero, leading to the zero contribution to the third order correlation function. The same is true for the second term in Eq. (V.18). Thus, the third order correlation function is equal to zero.

Similar analysis can be performed for any odd order correlation function: the corresponding product of matrix elements will be zero as there will only be an odd number of steps available in the step graphs. Hence, any odd order correlation function is equal to zero.

\section{Even order correlation functions}

Similarly one can consider higher order even correlation functions. It follows that, when averaging over phases, that nonzero contributions arise only by pairing integers belonging to complex conjugate exponentials (note that integers from different pairs may coincide as well, however, this 
falls within the remit of the pairing scheme, i.e. this case does not need to be considered separately). However, we find that the final expression does not contain a sum of products of only pair correlation functions, see Eq. (V.14), as there will be cross-terms as well.

To illustrate this point, consider the 4th order correlation function

$$
\begin{gathered}
\overline{\mathcal{R}_{i_{1}}\left(t_{1}\right) \mathcal{R}_{i_{2}}\left(t_{2}\right) \mathcal{R}_{i_{3}}\left(t_{3}\right) \mathcal{R}_{i_{4}}\left(t_{4}\right)} \\
=\sum_{m_{1} \neq n_{1}} \sum_{m_{2} \neq n_{2}} \sum_{m_{3} \neq n_{3}} \sum_{m_{4} \neq n_{4}} \overline{e^{i\left(\theta_{n_{1}}-\theta_{m_{1}}\right)} e^{i\left(\theta_{n_{2}}-\theta_{m_{2}}\right)} e^{i\left(\theta_{n_{3}}-\theta_{m_{3}}\right)} e^{i\left(\theta_{n_{4}}-\theta_{m_{4}}\right)}} \\
\times Z_{i_{1}}^{m_{1} n_{1}}\left(t_{1}\right) Z_{i_{2}}^{m_{2} n_{2}}\left(t_{2}\right) Z_{i_{3}}^{m_{3} n_{3}}\left(t_{3}\right) Z_{i_{4}}^{m_{4} n_{4}}\left(t_{4}\right)
\end{gathered}
$$

When pairing the phases, nine contributions emerge overall, see Fig. 2(c). They are split into two types of terms. Consider first the term associated with the pairing 1 in the Figure. It leads to the factor $\delta_{n_{1} m_{2}} \delta_{n_{2} m_{1}} \delta_{n_{3} m_{4}} \delta_{n_{4} m_{3}}$ after averaging and hence to the following contribution to the correlation function:

$$
\left[\sum_{n_{1} n_{2}} Z_{i_{1}}^{n_{1} n_{2}}\left(t_{1}\right) Z_{i_{2}}^{n_{2} n_{1}}\left(t_{2}\right)\right]\left[\sum_{n_{3} n_{4}} Z_{i_{3}}^{n_{3} n_{4}}\left(t_{3}\right) Z_{i_{4}}^{n_{4} n_{3}}\left(t_{4}\right)\right]=\overline{\mathcal{R}_{i_{1}}\left(t_{1}\right) \mathcal{R}_{i_{2}}\left(t_{2}\right)} \times \overline{\mathcal{R}_{i_{3}}\left(t_{3}\right) \mathcal{R}_{i_{4}}\left(t_{4}\right)}
$$

We see that this particular pairing scheme yields a product of two pair correlation functions, cf. Eq. (V.14).

There are two more pairing schemes, 6 and 9 in Fig. 2(c), which lead to a product of pair correlation functions as well. This can be seen e.g. by permuting pairs of numbers in the schemes. For instance, after permuting $2 \overline{2} \longleftrightarrow 3 \overline{3}$ in pairing scheme 6 , it becomes identical to the pairing scheme 1. Hence, the contribution of scheme 6 can be obtained from the above expression (V.19) by the permutation $i_{2}, t_{2} \longleftrightarrow i_{3}, t_{3}$.

The second type of terms is provided by the other six pairing schemes $2,3,4,5,7$, and 8 in Fig. 2(c). Scheme 2 yields the factor of $\delta_{n_{1} m_{2}} \delta_{n_{2} m_{4}} \delta_{n_{3} m_{1}} \delta_{n_{4} m_{3}}$ and hence the contribution

$$
\sum_{n_{1} n_{2} n_{3} n_{4}} Z_{i_{1}}^{n_{3} n_{1}}\left(t_{1}\right) Z_{i_{2}}^{n_{1} n_{2}}\left(t_{2}\right) Z_{i_{3}}^{n_{4} n_{3}}\left(t_{3}\right) Z_{i_{4}}^{n_{2} n_{4}}\left(t_{4}\right)
$$

to the correlation function. This term cannot be split into a simple product of two pair correlation functions since all four $Z$-coefficients are coupled. Other pairing schemes $3,4,5,7$ and 8 can be related to this one by an appropriate permutation of the pairs of numbers. For instance, pairing scheme 3 is brought into 2 by the permutation $4 \overline{4} \longleftrightarrow 3 \overline{3}$, and hence the final contribution of pairing 3 is obtained from Eq. (V.20) by the permutation $i_{4}, t_{4} \longleftrightarrow i_{3}, t_{3}$. The same can be done for all other pairing schemes belonging to this second type of pairings. 
Therefore, it is sufficient to consider just one pairing scheme of this type. We shall consider the scheme 2. First, we reorder terms in the product (V.20) of the $Z$-coefficients:

$$
Z_{i_{1}}^{n_{3} n_{1}}\left(t_{1}\right) Z_{i_{2}}^{n_{1} n_{2}}\left(t_{2}\right) Z_{i_{4}}^{n_{2} n_{4}}\left(t_{4}\right) Z_{i_{3}}^{n_{4} n_{3}}\left(t_{3}\right)
$$

This ensures the continuous flow of the state numbers, $n_{3} \rightarrow n_{1} \rightarrow n_{2} \rightarrow n_{4} \rightarrow n_{3}$, when reading from left to right. Hence, we can now turn to step graphs which start and end at the same state $n_{3}$. There are four $Z$-coefficients and hence we can make four steps overall; 2 steps up and 2 steps down would give nonzero contributions. There are 6 such step graphs possible, all shown in Fig. 4(b), any of them gives a nonzero contribution. The graphs in the Figure are arranged in pairs horizontally which provide contributions that are complex conjugate to each other. The top left graph in Fig. 4(b) is associated with the contribution

$$
\begin{gathered}
\sum_{\lambda} \sum_{n_{1} n_{2} n_{3} n_{4}} \delta_{n_{1} n_{4}} A_{n_{3} n_{1}} A_{n_{1} n_{2}} A_{n_{2} n_{1}} A_{n_{1} n_{3}} D_{i_{1} \lambda}^{*} D_{i_{2} \lambda}^{*} D_{i_{3} \lambda} D_{i_{4} \lambda}\left|\left\langle n_{1}\left|b_{\lambda}^{\dagger}\right| n_{3}\right\rangle\left\langle n_{2}\left|b_{\lambda}^{\dagger}\right| n_{1}\right\rangle\right|^{2} \\
=\sum_{\lambda} A_{\lambda}^{(a)} V_{i_{1} \lambda}\left(t_{1}\right) V_{i_{2} \lambda}\left(t_{2}\right) V_{i_{3} \lambda}\left(t_{3}\right) V_{i_{4} \lambda}\left(t_{4}\right) e^{-i \omega_{\lambda}\left(t_{1}+t_{2}-t_{3}-t_{4}\right)}
\end{gathered}
$$

where

$$
A_{\lambda}^{(a)}=\left(\frac{\hbar}{2 \omega_{\lambda}}\right)^{2} \sum_{n_{1} n_{2} n_{3}}\left|A_{n_{3} n_{1}} A_{n_{1} n_{2}}\left\langle n_{1}\left|b_{\lambda}^{\dagger}\right| n_{3}\right\rangle\left\langle n_{2}\left|b_{\lambda}^{\dagger}\right| n_{1}\right\rangle\right|^{2}
$$

is a positive real factor. Note that because of the matrix elements, the states in the triple sum above are constrained by the conditions: $n_{1} \neq n_{2}, n_{3}$.

The step graph on the right of the one we have just considered results in a complex conjugate contribution, leading therefore to the following real contribution from both these graphs:

$$
2 \sum_{\lambda} A_{\lambda}^{(a)} V_{i_{1} \lambda}\left(t_{1}\right) V_{i_{2} \lambda}\left(t_{2}\right) V_{i_{3} \lambda}\left(t_{3}\right) V_{i_{4} \lambda}\left(t_{4}\right) \cos \left(\omega_{\lambda}\left(t_{1}+t_{2}-t_{3}-t_{4}\right)\right)
$$

Similarly the other two pairs of the step graphs can be considered, resulting in the following contribuitons:

$$
2 \sum_{\lambda} A_{\lambda}^{(b)} V_{i_{1} \lambda}\left(t_{1}\right) V_{i_{2} \lambda}\left(t_{2}\right) V_{i_{3} \lambda}\left(t_{3}\right) V_{i_{4} \lambda}\left(t_{4}\right) \cos \left(\omega_{\lambda}\left(t_{1}-t_{2}-t_{3}+t_{4}\right)\right)
$$

is associated with the two graphs in the middle of Fig. 4(b), while

$$
2 \sum_{\lambda} A_{\lambda}^{(a)} V_{i_{1} \lambda}\left(t_{1}\right) V_{i_{2} \lambda}\left(t_{2}\right) V_{i_{3} \lambda}\left(t_{3}\right) V_{i_{4} \lambda}\left(t_{4}\right) \cos \left(\omega_{\lambda}\left(t_{1}-t_{2}+t_{3}-t_{4}\right)\right)
$$

is attributed to the two graphs at the bottom. Here

$$
A_{\lambda}^{(b)}=\left(\frac{\hbar}{2 \omega_{\lambda}}\right)^{2} \sum_{n_{1} n_{2}}\left|A_{n_{1} n_{2}}\left\langle n_{2}\left|b_{\lambda}^{\dagger}\right| n_{1}\right\rangle\right|^{4}
$$


The sum of the three contributions (V.21)-(V.23) corresponds to pairing scheme 2 in Fig. 2(c).

Other pairing schemes $3,4,5,7$ and 8 in the Figure are obtained by permuting indices $i_{j}$ and the times $t_{j}$ as was explained above. As can be seen from the formulae (V.21)-(V.23), each contribution is symmetric with respect to the permutations of the indices $i_{j}$, so only times need to be permuted. The final expression for the correlation function, containing contributions from all pairing schemes, becomes fully symmetric with respect to permutations of times. The correlation function depends on two unknown coefficients $A_{\lambda}^{(a)}$ and $A_{\lambda}^{(b)}$ for each normal mode $\lambda$ of the bath.

Hence, the 4th order correlation function, apart from the three terms corresponding to a product of all possible pair correlation functions, contains additional non-zero terms which cannot be represented as a product of pair correlations functions.

The method developed above can be applied without difficulty to higher (even) order correlation functions if necessary.

\section{E. Are the stochastic forces Gaussian?}

We conclude that the higher order correlation functions do not fully satisfy the properties of a Gaussian stochastic process: although correlation functions of any odd order are zero, even order correlation functions do not split solely into a sum of products of pair correlation functions; there are additional nonzero terms as well. This property of the stochastic forces poses a certain difficulty in numerical simulations as, at variance with the Gaussian stochastic forces of the classical case, a two-force correlation function is not sufficient for numerical simulations in the quantum case, i.e. higher order correlation functions need also be considered when generating the stochastic forces in actual numerical simulations. As the simplest approximation, one can assume that the stochastic forces in the $c$-number quantum GLE equations are Gaussian in which case only the lowest order (pair) correlation function suffices.

\section{SAMPLING OVER THE STOCHASTIC FIELD}

Because of the way the stochastic field has been introduced, exact results can only formally be obtained by averaging the calculated trajectories using different sampling of the stochastic field. This follows from the fact that we obtain the same equations of motion for the exact mean values $\left\langle\mathbf{x}_{1}\right\rangle_{t}=\operatorname{Tr}\left(\rho^{0} \widetilde{\mathbf{x}}_{1}(t)\right)$ after averaging over the stochastic field and after calculating the expectation values of the position operator $\mathbf{x}_{1}$ without the stochastic field in it, i.e. using directly the density 
matrix (IV.2).

Indeed, let us first perform the stochastic averaging of the equations of motion (IV.7). Since $\overline{\rho^{S}}=\rho^{0}$, the averaged mean values $\overline{\left\langle\mathbf{x}_{1}\right\rangle_{t}^{S}}=\operatorname{Tr}\left(\overline{\rho^{S}} \widetilde{\mathbf{x}}_{1}(t)\right)$ can be replaced with the exact ones, $\left\langle\mathbf{x}_{1}\right\rangle_{t}=\operatorname{Tr}\left(\rho^{0} \widetilde{\mathbf{x}}_{1}(t)\right)$. Next, since the stochastic average of the random force is zero, the equation for the mean values of the positions reads:

$$
\mathbf{M}_{11} \partial_{t}^{2}\left\langle\mathbf{x}_{1}\right\rangle_{t}=\mathbf{F}_{1}(t)-\int_{t_{0}}^{t} \mathbf{K}_{11}(t, \tau) \partial_{t}\left\langle\mathbf{x}_{1}\right\rangle_{\tau} d \tau
$$

It is easily checked that the same equation is obtained directly by performing quantum statistical average of the Heisenberg equations (III.11) using the exact density matrix $\rho^{0}$ of Eq. (IV.2). To this end, we multiply both sides of this equation by $\rho^{0}$ and take the trace over the whole system. The average of the random force operator $\mathbf{R}_{1}(t)$ reads

$$
\left\langle\mathbf{R}_{1}(t)\right\rangle_{t}=\operatorname{Tr}_{1}\left\langle\mathbf{R}_{1}(t)\right\rangle_{2}=-\mathbf{V}_{12}(t)\left[\dot{\boldsymbol{\Omega}}_{22}\left(t-t_{0}\right)\left\langle\mathbf{x}_{2}\right\rangle_{t}+\mathbf{\Omega}_{22}\left(t-t_{0}\right)\left\langle\mathbf{p}_{2}\right\rangle_{t}+\boldsymbol{\Pi}_{22}\left(t-t_{0}\right)\left\langle\mathbf{g}_{2}\right\rangle_{t}\right]
$$

where $\left\langle\mathbf{g}_{2}\right\rangle_{t}=\mathbf{V}_{2}\left(t_{0}\right)$ (see the definition (III.10)). Using Eq. (IV.2), we write:

$$
\left\langle\mathbf{x}_{2}\right\rangle_{t}=\operatorname{Tr}_{1} \sum_{n} \rho_{n}^{e q} \rho_{1}^{0}\left\langle\psi_{n}\left|\mathbf{x}_{2}\right| \psi_{n}\right\rangle=-\mathbf{D}_{22}^{-1} \operatorname{Tr}_{1}\left(\rho_{1}^{0} \mathbf{g}_{2}\right)=-\mathbf{D}_{22}^{-1}\left\langle\mathbf{g}_{2}\right\rangle_{t}
$$

and $\left\langle\mathbf{p}_{2}\right\rangle_{t}=\mathbf{0}$, cf. the derivation of Eqs. (V.7) and (V.8), and we obtain $\left\langle\mathbf{R}_{1}(t)\right\rangle_{t}=\mathbf{0}$. Hence, multiplying both sides of Eq. (III.11) by $\rho^{0}$, taking the trace over the whole system and using the fact that $\operatorname{Tr}\left(\rho^{0} \widetilde{\mathbf{u}}_{1}\right)=\mathbf{0}$, we arrive at the same Eq. (VI.1) for the exact mean values.

We conclude that exact results can only formally be obtained by averaging the calculated trajectories over different realisations of the stochastic field.

\section{THE CLOSURE RELATIONSHIP}

Until now we have demonstrated that there exists a class of cQGLEs of the form similar to the classical GLE and containing a generally non-Gaussian random forces. If one assumes that the forces are approximately Gaussian, then the pair correlation function is sufficient to generate them. However, in order to know the pair correlation function, according to Eq. (V.15), one need to determine the unknown amplitudes $A_{\lambda}$. Here we shall propose a heuristic argument that yields an explicit expression for $A_{\lambda}$ with the correct classical limit. It is based on an assumption that the correlation function of the random force does not depend on the order in which the averages are taken. 
Eq. (V.15) was derived with the trace over both regions (the quantum statistical average) taken first to yield $\boldsymbol{R}_{1}(t)=\operatorname{Tr}\left(\mathbf{R}_{1}(t)\right)$, and only after that the average over the stochastic field was applied when calculating the correlation function over the stochastic field of the phases. However, the calculation can also be done in a different order: first, we average with respect to the stochastic field (which results in the initial density matrix since $\overline{\rho^{S}} \equiv \rho^{0}$ ), and only then the trace with the averaged density matrix $\rho^{0}$ is performed. Our hypothesis states that the correlation functions calculated in both ways must coincide. Since the correlation function (V.15) is symmetric with respect to the time permutation, $t \leftrightarrow t^{\prime}$ (and is real), the required condition (which we shall call the closure relationship) is:

$$
\overline{\mathcal{R}_{1}(t) \boldsymbol{\mathcal { R }}_{1}^{T}\left(t^{\prime}\right)}=\frac{1}{2}\left[\left\langle\mathbf{R}_{1}(t) \mathbf{R}_{1}^{\dagger}\left(t^{\prime}\right)\right\rangle_{a v}+\left\langle\mathbf{R}_{1}\left(t^{\prime}\right) \mathbf{R}_{1}^{\dagger}(t)\right\rangle_{a v}\right]
$$

Here the operator $\mathbf{R}_{1}(t)$ is defined by Eq. (III.14). In calculating the averages $\langle\ldots\rangle_{a v}=$ $\operatorname{Tr}_{1} \operatorname{Tr}_{2}\left(\rho_{0} \ldots\right)=\operatorname{Tr}_{1}\langle\ldots\rangle_{2}$, we use the explicit expression (IV.2) for $\rho^{0}$.

When calculating the whole trace $\langle\ldots\rangle_{a v}$, it is convenient to perform the trace over the bath first:

$$
\begin{gathered}
\left\langle\mathbf{R}_{1}(t) \mathbf{R}_{1}^{\dagger}\left(t^{\prime}\right)\right\rangle_{2}=\rho_{1}^{0} \sum_{m} \rho_{m}^{e q}\left\langle\psi_{m}\left|\mathbf{R}_{1}(t) \mathbf{R}_{1}^{\dagger}\left(t^{\prime}\right)\right| \psi_{m}\right\rangle \\
=\rho_{1}^{0} \sum_{m} \rho_{m}^{e q}\left\langle m\left|\mathcal{U}^{\dagger} \mathbf{R}_{1}(t) \mathbf{R}_{1}^{\dagger}\left(t^{\prime}\right) \mathcal{U}\right| m\right\rangle=\rho_{1}^{0} \sum_{m} \rho_{m}^{e q}\left\langle m\left|\widetilde{\mathbf{R}}_{1}(t) \widetilde{\mathbf{R}}_{1}^{T}\left(t^{\prime}\right)\right| m\right\rangle
\end{gathered}
$$

where $\widetilde{\mathbf{R}}_{1}(t)=\mathcal{U}^{\dagger} \mathbf{R}_{1}(t) \mathcal{U}$. To calculate the latter force operator, it is useful first to simplify the expression for the force. From Eq. (III.14)

$$
\begin{gathered}
\mathbf{R}_{1}(t)=-\sum_{\lambda} \sqrt{\frac{\hbar}{2 \omega_{\lambda}}} \mathbf{V}_{12}(t)\left[\left(\dot{\Omega}_{22}\left(t-t_{0}\right)+i \omega_{\lambda} \Omega_{22}\left(t-t_{0}\right)\right) e_{\lambda} b_{\lambda}^{\dagger}+\left(\dot{\Omega}_{22}\left(t-t_{0}\right)-i \omega_{\lambda} \Omega_{22}\left(t-t_{0}\right)\right) e_{\lambda} b_{\lambda}\right] \\
-\mathbf{V}_{12}(t) \boldsymbol{\Pi}_{22}\left(t-t_{0}\right) \mathbf{g}_{2}
\end{gathered}
$$

Using Eqs. (II.8) and (II.9), we find that

$$
\left(\dot{\Omega}_{22}\left(t-t_{0}\right)+i \omega_{\lambda} \Omega_{22}\left(t-t_{0}\right)\right) \mathbf{e}_{\lambda}=\mathbf{e}_{\lambda} e^{i \omega_{\lambda}\left(t-t_{0}\right)}
$$

so that we finally obtain for the force operator an expression:

$$
\mathbf{R}_{1}(t)=\sum_{\lambda}\left[\mathbf{D}_{1 \lambda}(t) b_{\lambda}^{\dagger}+\mathbf{D}_{1 \lambda}^{*}(t) b_{\lambda}\right]-\mathbf{V}_{12}(t) \boldsymbol{\Pi}_{22}\left(t-t_{0}\right) \mathbf{g}_{2}
$$

where the elements of the vector $\mathbf{D}_{1 \lambda}(t)=\left(D_{i \lambda}, i \in 1\right)$ are given by Eq. (V.11). 
Then using the fact that $\mathcal{U}^{\dagger} b_{\lambda} \mathcal{U}=b_{\lambda}+\gamma_{\lambda}$ and $\mathcal{U}^{\dagger} b_{\lambda}^{\dagger} \mathcal{U}=b_{\lambda}^{\dagger}+\gamma_{\lambda}$, we easily obtain:

$$
\widetilde{\mathbf{R}}_{1}(t)=\sum_{\lambda}\left[\mathbf{D}_{1 \lambda}(t) b_{\lambda}^{\dagger}+\mathbf{D}_{1 \lambda}^{*}(t) b_{\lambda}\right]
$$

We see that the third term in Eq. (VII.2) disappears completely, which renders the tilde-force operator $\widetilde{\mathbf{R}}_{1}(t)$ to be independent of the displacement vector $\mathbf{u}_{1}$. Hence, the trace over the open system is trivially calculated resulting in the following expression for the correlation function:

$$
\left\langle\mathbf{R}_{1}(t) \mathbf{R}_{1}^{\dagger}\left(t^{\prime}\right)\right\rangle_{a v}=\sum_{m} \rho_{m}^{e q}\left\langle m\left|\widetilde{\mathbf{R}}_{1}(t) \widetilde{\mathbf{R}}_{1}^{T}\left(t^{\prime}\right)\right| m\right\rangle=\left\langle\widetilde{\mathbf{R}}_{1}(t) \widetilde{\mathbf{R}}_{1}^{T}\left(t^{\prime}\right)\right\rangle_{2}^{e q}
$$

This is nothing but the trace over the bath using its equilibrium density matrix. Therefore, from Eq. (VII.3) it is clear that, upon multiplication of the tilda-forces, only products of annihilation and creation operators will contribute:

$$
\left\langle\widetilde{\mathbf{R}}_{1}(t) \widetilde{\mathbf{R}}_{1}^{T}\left(t^{\prime}\right)\right\rangle_{2}^{e q}=\sum_{\lambda \lambda^{\prime}}\left\{\left[\mathbf{D}_{1 \lambda}(t) \mathbf{D}_{1 \lambda^{\prime}}^{\dagger}\left(t^{\prime}\right)\right]\left\langle b_{\lambda}^{\dagger} b_{\lambda^{\prime}}\right\rangle_{2}^{e q}+\left[\mathbf{D}_{1 \lambda}(t) \mathbf{D}_{1 \lambda^{\prime}}^{\dagger}\left(t^{\prime}\right)\right]^{*}\left\langle b_{\lambda} b_{\lambda^{\prime}}^{\dagger}\right\rangle_{2}^{e q}\right\}
$$

Since

$$
\mathbf{D}_{1 \lambda}(t) \mathbf{D}_{1 \lambda^{\prime}}^{\dagger}\left(t^{\prime}\right)=\mathbf{V}_{12}(t)\left[\frac{\hbar}{2 \omega_{\lambda}} \mathbf{e}_{\lambda} \mathbf{e}_{\lambda}^{T} e^{i \omega_{\lambda}\left(t-t^{\prime}\right)}\right] \mathbf{V}_{21}\left(t^{\prime}\right)
$$

and $\left\langle b_{\lambda}^{\dagger} b_{\lambda^{\prime}}\right\rangle_{2}^{e q}=\left\langle b_{\lambda} b_{\lambda^{\prime}}^{\dagger}\right\rangle_{2}^{e q}-\delta_{\lambda \lambda^{\prime}}=\delta_{\lambda \lambda^{\prime}} \bar{n}_{\lambda}=\delta_{\lambda \lambda^{\prime}}\left(e^{\beta \hbar \omega_{\lambda}}-1\right)^{-1}$, we obtain:

$$
\left\langle\mathbf{R}_{1}(t) \mathbf{R}_{1}^{\dagger}\left(t^{\prime}\right)\right\rangle_{a v}=\mathbf{V}_{12}(t)\left[\sum_{\lambda} \frac{\hbar}{\omega_{\lambda}}\left(\bar{n}_{\lambda}+\frac{1}{2}\right) \mathbf{e}_{\lambda} \mathbf{e}_{\lambda}^{T} \cos \left(\omega_{\lambda}\left(t-t^{\prime}\right)\right)-\frac{i \hbar}{2} \boldsymbol{\Omega}_{22}\left(t-t^{\prime}\right)\right] \mathbf{V}_{21}\left(t^{\prime}\right)
$$

where Eq. (II.8) was also used. Note that the second term is purely imaginary and is antisymmetric with respect to the time permutation. This term disappears when using the above expression in the closure relationship, Eq. (VII.1), which yields:

$$
\overline{\mathcal{R}_{1}(t) \mathcal{R}_{1}^{T}\left(t^{\prime}\right)}=\mathbf{V}_{12}(t)\left[\sum_{\lambda} \frac{\hbar}{\omega_{\lambda}}\left(\bar{n}_{\lambda}+\frac{1}{2}\right) \mathbf{e}_{\lambda} \mathbf{e}_{\lambda}^{T} \cos \left(\omega_{\lambda}\left(t-t^{\prime}\right)\right)\right] \mathbf{V}_{21}\left(t^{\prime}\right)
$$

The expression on the right hand side has the same form as expression (V.15), where we used a different ordering for the averages (i.e. first the quantum statistical average and then the stochastic average). Comparing these two expressions, we obtain the following formula for the amplitudes we have been looking for:

$$
A_{\lambda}=\frac{\hbar}{\omega_{\lambda}}\left(\bar{n}_{\lambda}+\frac{1}{2}\right)=\frac{\hbar}{2 \omega_{\lambda}} \operatorname{coth}\left(\frac{1}{2} \beta \hbar \omega_{\lambda}\right)
$$


It can be seen that this expression for the amplitude tends to the correct classical limit, $A_{\lambda} \rightarrow$ $1 / \beta \omega_{\lambda}^{2}$, when either $\hbar \rightarrow 0$ or $\beta \rightarrow 0$. It is highly encouraging that the same form of the pair correlation function has been obtained by the two methods. Moreover, the second method enabled us to propose an explicit expression for the previously unknown amplitudes $A_{\lambda}$. The result we obtained basically coincides with the expression from [21, 22, 25, 32] for the correlation function that was used in [45-48] without a proper justification (and for a more simplified Hamiltonian).

Unfortunately, an analogous procedure does not seem to exist for higher order correlation functions. This is because an average of a product of the force correlations functions,

$$
\left\langle R_{i_{1}}\left(t_{1}\right) R_{i_{2}}\left(t_{2}\right) R_{i_{3}}\left(t_{3}\right) \ldots\right\rangle_{a v}=\left\langle\widetilde{R}_{i_{1}}\left(t_{1}\right) \widetilde{R}_{i_{2}}\left(t_{2}\right) \widetilde{R}_{i_{3}}\left(t_{3}\right) \ldots\right\rangle_{2}^{e q}
$$

is given by the quantum-statistical average over the equilibrium bath, as given above, and hence the Wick's theorem can be used to calculate it. An appropriate calculation shows that odd order averages are all zero, but even order ones split into a sum of products of only pair correlations (VII.4). Hence, the extra terms we encountered in Section V D do not appear at all in the averages (VII.8). So the question of obtaining unknown amplitudes in the extra terms in the even order

correlation functions (as e.g. $A_{\lambda}^{(a)}$ and $A_{\lambda}^{(b)}$ from Section V D), still remains open. One must expect that these extra terms that cannot be represented as a product of pair correlation functions, must tend to zero in the classical limit of $\hbar, \beta \rightarrow 0$. This would ensure that in the classical limit the random forces correspond to a Gaussian stochastic process.

\section{CONCLUSIONS}

Concluding, in this paper we offer a derivation of a fully quantum $c$-number GLE which is a self-contained equation for the expectation values of the positions of atoms in the open system. Our method is based on a rather general Hamiltonian of the combined system (the open system and bath) which possesses a surprising similarity with its classical analogue [17]: an identical friction kernel and a similar structure of the random force autocorrelation function. Our derivation is based on the harmonisation approximation whereby a harmonic expansion is made around the exact instantaneous mean values of the positions of atoms in the open system which evolve in time. A possible direction for future research is to go beyond this approximation e.g. along the lines proposed in $[29,46]$. No product approximation was assumed in our treatment for the initial density matrix of the combined system as done e.g. in the Feynman-Vernon method [39, 42] and some of the traditional quantum (operator) GLE approaches [32]. Our equations contain 
non-Gaussian stochastic forces, which have zero mean and appropriate correlation functions, and perform as a coloured noise. The simplest pair correlation function has the same functional form as in the classical case [17], but contains amplitudes $A_{\lambda}$ which may depend on the initial preparation of the open system and, of course, on the temperature.

It is also shown that after sampling over many trajectories due to different realisations of the stochastic field, our approach converges to the exact trajectory for the mean values $\left\langle x_{1}\right\rangle_{t}$ of the open system atomic positions.

In the first approximation, stochastic forces may be considered as Gaussian. To offer a practical computational scheme, an explicit expression for the amplitudes of the pair correlation function was offered. It is based on a conjecture that no matter in which order the stochastic and statistical averages are taken when calculating the random force autocorrelation function, the same result is to be expected. The obtained expression for the amplitudes has the correct classical limit.

Our method sets a foundation for a practical "classical"-like computational technique, which could be used for calculating atomic trajectories in an open system under arbitrary non-equilibrium conditions - these would be fully quantum MD simulations for mean atomic positions. Note that quantum MD simulations based on path integrals $[56,57]$ are designed only for thermodynamic equilibrium.

One may ask why perform quantum MD simulations with stochastic forces with subsequent averaging over many realisations of the stochastic field instead of solving directly the equations for the mean atomic positions that do not contain the stochastic force? There are at least two advantages in using stochastic methods: (i) stochastic equations of motion give access to fluctuations of atomic trajectories from the mean trajectory, and (ii) there exist powerful numerical techniques for solving stochastic differential equations with the memory and random forces [18-20] which can be exploited (assuming that stochastic forces are Gaussian). Of course, further work is needed in designing a computational scheme which accounts for the non-Gaussian character of the random force.

\section{ACKNOWLEDGEMENTS}

We acknowledge financial support from the UK EPSRC under Grant No. EP/J019259/1. HN, CDL and LK acknowledge the stimulating research environment provided by the EPSRC Centre for Doctoral Training in Cross-Disciplinary Approaches to Non-Equilibrium Systems (CANES, $\mathrm{EP} / \mathrm{L} 015854 / 1)$. We would also like to acknowledge a financial support of the COST action 
MP1303 "Understanding and controlling nano and mesoscale friction" for facilitating valuable discussions.

[1] H.-P. Breuer and F. Petruccione, The Theory of Open Quantum Systems, Oxford Univ. Press, 2007.

[2] U. Weiss, Quantum Dissipative System, World Scientific, Singapore, 2012.

[3] I. Szlufarska, R. K. Kalia, A. Nakano, and P. Vashishta, A molecular dynamics study of nanoindentation of amorphous silicon carbide, J. Appl. Phys. 102, 023509 (2007).

[4] P. R. Barry, P. Y. Chiu, S. S. Perry, W. G. Sawyer, S. R. Phillpot, and S. B. Sinnott, The effect of normal load on polytetrafluoroethylene tribology, J.Phys.: Condens. Matter 21, 144201 (2009).

[5] Christian D. Lorenz, Michael Chandross, and Gary S. Grest, Large scale molecular dynamics simulations of vapor phase lubrication for MEMS, J. Adh. Sci. Tech. 24, 2453 (2010).

[6] T. Trevethan and L. Kantorovich, Stochastic mechanism of energy dissipation in noncontact atomic force microscopy studied using molecular dynamics with Langevin boundary conditions, Phys. Rev. B, 70, $115411(2004)$.

[7] J. R. Kermode, T. Albaret, D. Sherman, N. Bernstein, P. Gumbsch, M. C. Payne, G. Csányi, and A. De Vita, Low-speed fracture instabilities in a brittle crystal, Nature 455, 1224 (2008).

[8] Oleg A. Mazyar and William L. Hase, Dynamics and kinetics of heat transfer at the interface of model diamond 111 nanosurfaces, J. Phys. Chem. A 110, 526 (2006).

[9] M. Hu, P. Keblinski, J.-S. Wang, and N. Raravikar, Interfacial thermal conductance between silicon and a vertical carbon nanotube, J. Appl. Phys. 104, 083503 (2008).

[10] A. Dhar and D. Roy, Heat transport in harmonic lattices, J. Stat. Phys. 125, 801 (2008).

[11] Jiuning Hu, Xiulin Ruan, and Yong P. Chen, Thermal conductivity and thermal rectification in graphene nanoribbons: A molecular dynamics study, Nano Lett. 9, 2730 (2009).

[12] Jing Guo, Bin Wen, Roderick Melnik, Shan Yao, and Tingju Li, Geometry and temperature dependent thermal conductivity of diamond nanowires: A non-equilibrium molecular dynamics study, Physica E 43, 155 (2010).

[13] Lin Hu, Tapan Desai, and Pawel Keblinski, Determination of interfacial thermal resistance at the nanoscale, Physical Review B 83, 195423 (2011).

[14] Paranjothy Manikandan, Jeffrey A. Carter, Dana D. Dlott, and William L. Hase, Effect of carbon chain length on the dynamics of heat transfer at a gold/hydrocarbon interface: Comparison of 
simulation with experiment, J. Phys. Chem. C 115, 9622 (2011).

[15] W.-D. Hsu, S. Tepavcevic, L. Hanley, and S. B. Sinnott, Mechanistic studies of surface polymerization by ion-assisted deposition, J. Phys. Chem. C 111, 4199 (2007).

[16] R. Zwanzig, Nonequilibrium Statistical Mechanics, Oxford University Press, 2001.

[17] L. N. Kantorovich, Generalized Langevin equation for solids: I Rigorous derivation and main properties, Phys. Rev. B 78, 094304 (2008).

[18] L. Stella, C. D. Lorenz, and L. Kantorovich, Generalized Langevin equation: An efficient approach to nonequilibrium molecular dynamics of open systems, Phys. Rev. B 89, 134303 (2014).

[19] H. Ness, L. Stella, C. D. Lorenz, and L. Kantorovich, Applications of the generalized Langevin equation: Towards a realistic description of the baths, Phys. Rev. B 91, 014301 (2015).

[20] H. Ness, A. Genina, L. Stella, C. D. Lorenz, and L. Kantorovich, Nonequilibrium processes from generalised Langevin equations: realistic nanoscale systems connected to two thermal baths, Phys. Rev. B 93, 174303 (2016).

[21] G. W. Ford, M. Kac, and P. Mazur, Statistical mechanics of assemblies of coupled oscillator, J. Math. Phys. 6, 504 (1965).

[22] Rafael Benguria and Mark Kac, Quantum Langevin equation, Phys. Rev. Lett. 46, 1 (1981).

[23] Katja Lindenberg and Bruce J. West, Statistical properties of quantum systems: The linear oscillator, Phys. Rev. A 30, 568 (1984).

[24] E. Cortes, B. J. West, and K. Lindenberg, On the generalised Langevin equation: classical and quantum mechanical, J. Chem. Phys. 82, 2708 (1985).

[25] G. W. Ford and M. Kac, On the quantum Langevin equation, J. Stat. Phys. 46, 803 (1987).

[26] G. W. Ford, J. T. Lewis, and R. F. O'Connell, Quantum Langevin equation, Phys. Rev. B 37, 4419 (1988).

[27] C. W. Gardiner, Quantum noise and quantum Langevin equations, IBM J. Res. Develop. 32, 127 (1988).

[28] N. G. van Kampen, Derivation of the quantum Langevin equation, J. Molec. Liquids 71, 97 (1997).

[29] H. K. McDowell, Quantum generalized Langevin equation: Explicit inclusion of nonlinear system dynamics, J. Chem. Phys. 112, 6971 (2000).

[30] Th. M. Nieuwenhuizen and A. E. Allahverdyan, Statistical thermodynamics of quantum Brownian motion: Construction of perpetuum mobile of the second kind, Phys. Rev. E 66, 036102 (2002). 
[31] Yi Jing Yan and Rui Xue Xu, Quantum mechanics of dissipative systems, Annu. Rev. Phys. Chem. 56, 187 (2005).

[32] C. W. Gardiner and P. Zoller, Quantum noise, Springer, 2010.

[33] L. Ferialdi and D. Dürr, Progress towards an effective non-Markovian description of a system interacting with a bath, Phys. Rev. A 91, 042130 (2015).

[34] D. Segal and A. Nitzan, Heating in current carrying molecular junctions, J. Chem. Phys. 117, 3915 (2002).

[35] D. Segal, A. Nitzan, and P. Hanggi, Thermal conductance through molecular wires, J. Chem. Phys. 119, 6840 (2003).

[36] A. Ozpineci and S. Ciraci, Quantum effects of thermal conductance through atomic chains, Phys. Rev. B 63, 125415 (2001).

[37] J.-S. Wang, J. Wang, and N. Zeng, Nonequilibrium greens function approach to mesoscopic thermal transport, Phys. Rev. B 74, 033408 (2006).

[38] M. Galperin, A. Nitzan, and M. A. Ratner, Heat conduction in molecular transport junctions, Phys. Rev. B 75, 155312 (2007).

[39] R. P. Feynman and F. L. Vernon, The theory of a general quantum system interacting with a linear dissipative system, Ann. Phys. 24, 118 (1963).

[40] K. L. Sebastian, The classical description of scattering from a quantum system, Chem. Phys. Lett. 81, 14 (1981).

[41] U. Eckern, W. Lehr, A. Menzel-Dorwarth, F. Pelzer, and A. Schmid, The quasiclassical langevin equation and its application to the decay of a metastable state and to quantum fluctuations, J. Stat. Phys. 59, 885 (1990).

[42] H. Kleinert and S.V. Shabanov, Quantum Langevin equation form forward-backward path integral, Phys. Lett. A 200, 224 (1995).

[43] J.-S. Wang, Quantum thermal transport from classical molecular dynamics, Phys. Rev. Lett. 99, 160601 (2007).

[44] H. Dammak, Y. Chalopin, M. Laroche, M. Hayoun, and J.-J. Greffet, Quantum thermal bath for molecular dynamics simulation, Phys. Rev. Lett. 103, 190601 (2009).

[45] D. Banerjee, B. C. Bag, S. K. Banik, and D. S. Ray, Approach to quantum kramers equation and barrier crossing dynamics, Phys. Rev. E 65, 021109 (2002). 
[46] D. Banerjee, S. K. Banik, B. C. Bag, and D. S. Ray, Quantum Kramers equation for energy diffusion and barrier crossing dynamics in the low-friction regime, Phys. Rev. E 66, 051105 (2002).

[47] S. K. Banik, B. C. Bag, and D. S. Ray, Generalized quantum Fokker-Planck, diffusion, and Smoluchowski equations with true probability distribution functions, Phys. Rev. E 65, 051106 (2002).

[48] D. Banerjee, B. C. Bag, S. K. Banika, and D. S. Ray, Solution of quantum Langevin equation: Approximations, theoretical and numerical aspects, J. Chem. Phys. 120, 8960 (2004).

[49] In Refs. [45-48] the Heisenberg operator equations of motion for the open system are first quantumaveraged using a predefined state of the whole system. The chosen wavefunction of this state is an Ansatz containing a product of an arbitrary state of the open system and a product of coherent states of each harmonic oscillator of the bath; no justification of why this particular product of states has been taken is provided. Then, they take a quantum-mechanical average of the operator equation of motion (instead of the quantum statistical-mechanical averaging), thereby arriving at a $c$-number Langevin equation. Finally, the correlation function of the random force is defined using a Gaussianlike Wigner distribution for a set of independent shifted harmonic oscillators. Again, there is no clear justification of why one should use such a procedure.

[50] A. O. Caldeira and A. J. Leggett, Path integral approach to quantum Brownian motion, Physics A 121, 587 (1983).

[51] D. Zubarev, V. Morozov, and G. Röpke, Statistical mechanics of nonequilibrium processes. Vol. 1: Basic concepts, kinetic theory, Akademie verlag, Berlin, 1996.

[52] A. P. Horsfield, D. R. Bowler, A. J. Fisher, T. N. Todorov, and C. G. Sánchez, Correlated electron ion dynamics: the excitation of atomic motion by energetic electrons, J. Phys.: Condens. Matter 17, 4793-4812 (2005).

[53] In particular, a very good agreement between the exact solution of the Schrödinger equation and a converged CEID calculation withing the harmonisation approximation has been demonstrated in L. Stella, M. Meister, A. J. Fisher and A. P. Horsfield, Robust nonadiabatic molecular dynamics for metals and insulators, J. Chem. Phys. 127, 214104 (2007).

[54] P. Gaspard and M. Nagaoka, Non-Markovian stochastic Schrödinger equation, J. Chem. Phys. 111, 5676 (1999).

[55] H. Risken, The Fokker-Planck equation. Methods of solution and applications, Springer-Verlag, Berlin, 2nd edition, 1989. 
[56] S. Habershon, D. E. Manolopoulos, T. E. Markland, and T. F. Miller III, Ring-polymer molecular dynamics: Quantum effects in chemical dynamics from classical trajectories in an extended phase space, Annu. Rev. Phys. Chem. 64, 387-413 (2013).

[57] C. P. Herrero and R. Ramírez, Path-integral simulation of solids, J. Phys.: Condens. Matter 26, 233201 (2014). 